\title{
ON THE MASSES OF GALAXIES IN THE LOCAL UNIVERSE
}

\author{
Edward N. Taylor ${ }^{1,2}$, Marijn Franx ${ }^{1}$, Jarle Brinchmann ${ }^{1}$, Arjen van der Wel ${ }^{3}$, And Pieter G. Van Dokkum ${ }^{4}$ \\ ${ }^{1}$ Sterrewacht Leiden, Leiden University, NL-2300 RA Leiden, The Netherlands; ent @ strw.leidenuniv.nl \\ ${ }^{2}$ School of Physics, The University of Melbourne, Parkville 3010, Australia \\ ${ }^{3}$ Max Planck Institut für Astronomie, D-69117 Heidelberg, Germany \\ ${ }^{4}$ Department of Astronomy, Yale University, New Haven, CT 06520-8101, USA \\ Received 2010 March 25; accepted 2010 June 11; published 2010 September 15
}

\begin{abstract}
We compare estimates of stellar mass, $M_{*}$, and dynamical mass, $M_{\mathrm{d}}$, for a sample of galaxies from the Sloan Digital Sky Survey. Under the assumption of dynamical homology (i.e., $\tilde{M}_{\mathrm{d}} \sim \sigma_{0}^{2} R_{\mathrm{e}}$, where $\sigma_{0}$ is the central velocity dispersion and $R_{\mathrm{e}}$ is the effective radius), we find a tight but strongly nonlinear relation between the two mass estimates: the best-fit relation is $M_{*} \propto \tilde{M}_{\mathrm{d}}^{0.73}$, with an observed scatter of 0.15 dex. We also find that, at fixed $M_{*}$, the ratio $M_{*} / \tilde{M}_{\mathrm{d}}$ depends strongly on galaxy structure, as parameterized by the Sérsic index, $n$. The size of the differential effect is on the order of 0.6 dex across $2<n<10$. The apparent $n$-dependence of $M_{*} / \tilde{M}_{\mathrm{d}}$ is qualitatively and quantitatively similar to expectations from simple, spherical and isotropic dynamical models, indicating that assuming homology gives the wrong dynamical mass. To explore this possibility, we have also derived dynamical mass estimates that explicitly account for differences in galaxies' structures. Using this "structure-corrected" dynamical mass estimator, $M_{\mathrm{d}, n}$, the best-fit relation is $M_{*} \propto M_{\mathrm{d}, n}^{0.92 \pm 0.01( \pm 0.08)}$ with an observed scatter of $0.13 \mathrm{dex}$. While the data are thus consistent with a linear relation, they do prefer a slightly shallower slope. Further, we see only a small residual trend in $M_{*} / M_{\mathrm{d}, n}$ with $n$. We find no statistically significant systematic trends in $M_{*} / M_{\mathrm{d}, n}$ as a function of observed quantities (e.g., apparent magnitude, redshift), or as a function of tracers of stellar populations (e.g., $\mathrm{H} \alpha$ equivalent width, mean stellar age), nor do we find significantly different behavior for different kinds of galaxies (i.e.,central versus satellite galaxies, emission versus non-emission galaxies). At $99 \%$ confidence, the net differential bias in $M_{*} / M_{\mathrm{d}, n}$ across a wide range of stellar populations and star formation activities is $\lesssim 0.12$ dex $(\approx 40 \%)$. The very good agreement between stellar mass and structure-corrected dynamical mass strongly suggests, but does not unambiguously prove, that (1) galaxy non-homology has a major impact on dynamical mass estimates, and (2) there are no strong systematic biases in the stellar mass-to-light ratios derived from broadband optical spectral energy distributions. Further, accepting the validity of both our stellar and dynamical mass estimates, these results suggest that the central dark-to-luminous mass ratio has a relatively weak mass dependence, but a very small scatter at fixed mass.
\end{abstract}

Key words: galaxies: fundamental parameters - galaxies: kinematics and dynamics - galaxies: stellar content galaxies: structure

Online-only material: color figures

\section{INTRODUCTION}

Techniques for estimating galaxies' stellar masses are a crucial tool for understanding galaxies and their evolution. There are tight and well-defined correlations between stellar mass and many other important global properties like color, size, structure, metallicity, star formation activity, and environment (see, e.g., Kauffmann et al. 2003b; Shen et al. 2003; Blanton et al. 2005a; Gallazzi et al. 2006). Given a galaxy's stellar mass, $M_{*}$, it is thus possible to predict a wide variety of global properties with considerable accuracy. In this sense, stellar mass appears to be a key parameter in determining (or at least describing) a galaxy's current state of evolution. Moreover, since the growth of stellar mass (cf. absolute luminosity, color, etc.) is relatively slow and approximately monotonic, stellar mass is a particularly useful parameter for quantifying galaxy evolution.

Stellar mass estimates, whether derived from spectroscopic or photometric spectral energy distributions (SEDs), are plagued by a variety of random and systematic errors. These include a generic degeneracy between mean stellar age, metallicity, and dust obscuration. It is typical to make the simplifying assumptions that galaxies' stellar populations can be described en masse (i.e.,neglecting age/metallicity gradients and complex dust geometries) and that galaxies' complex star formation histories can be described parametrically. It is rare to attempt to account for active galactic nucleus (AGN) emission. The stellar initial mass function (IMF), including its universality or otherwise, remains a major "known unknown." Then there is the complication that different wavelengths probe different aspects of the stellar population; the inclusion of rest-frame UV or NIR data can thus, in principle and in practice, have a large impact on the estimated stellar mass. These effects are compounded by uncertainties in the stellar evolution models themselves. A topical example is the importance of NIR-luminous thermally pulsating asymptotic giant branch (TP-AGB) stars: for the same data and stellar population parameters, the use of the Bruzual \& Charlot (2003) or Maraston (2005) models can change the derived value of $M_{*}$ by a factor of 3 for galaxies that host young $(\lesssim 1 \mathrm{Gyr})$ stars, but only if rest-frame NIR data are included (van der Wel et al. 2006; Kannappan \& Gawiser 2007). Conroy et al. (2009) have argued that the total random uncertainties in $M_{*}$ are on the order of $\sim 0.3$ dex for galaxies at $z \sim 0$.

For these reasons, it is essential to devise some way of assessing the quality of stellar mass estimates through comparison to some other fiducial mass estimate- this is the primary motivation for the present paper. Specifically, using a number of 
the publicly available "value added" catalogs of the Sloan Digital Sky Survey (SDSS; York et al. 2000; Strauss et al. 2002), we will compare stellar mass estimates to total mass estimates derived from galaxy dynamics.

From the outset, we note that a difference between two quantities shows only that: a difference. With no definitive standard to use as a basis for comparison, the best that we can hope for is consistency between the two mass estimates. Further, if and when there are differences, it is impossible to unambiguously identify where the "fault" lies-or even if there is indeed a fault. For example, it is likely that the ratio between stellar and total mass varies as a function of mass, and/or some other global property(ies). We will also explore this issue in some detail.

This kind of comparison has been done for SDSS galaxies by Drory et al. (2004), who considered both stellar mass estimates derived from the SDSS spectra, as described by Kauffmann et al. (2003a), and those derived using SED-fitting techniques that are commonly used at for higher-redshift studies. These authors find a relatively tight correlation between the two stellar mass estimates, with a mild systematic bias depending on $\mathrm{H} \alpha$ equivalent width (EW). This bias suggests a potential problem with the stellar mass estimates as a function of specific star formation rate (SSFR). Further, both stellar mass estimates correlated well with the simple dynamical mass estimate, $\tilde{M}_{\mathrm{d}}$ (defined below), but showed a clear trend in $M_{*} / \tilde{M}_{\mathrm{d}}$ with mass, such that less massive galaxies had higher values of $M_{*} / \tilde{M}_{\mathrm{d}}$. Padmanabhan et al. (2004) used the mass dependence of $M_{*} / \tilde{M}_{\mathrm{d}}$ to argue for an increasing stellar-to-dark mass ratio for elliptical galaxies with higher masses, as did Gallazzi et al. (2005). Both Rettura et al. (2006) and van der Wel et al. (2006) have performed similar comparisons for $z \lesssim 1$ galaxies, with similar conclusions.

It is common practice to derive a simple dynamical mass estimate based on the velocity dispersion, $\sigma_{0}$, and the effective radius, $R_{\mathrm{e}}$, via the scalar virial theorem:

$$
G \tilde{M}_{\mathrm{d}} \approx k \sigma_{0}^{2} R_{\mathrm{e}} .
$$

(This is the dynamical mass estimator used for each of the studies cited in the previous paragraph.) The constant $k$ is usually assumed to be in the range $3-5$, and is intended to account for the "degree of virialization," including the effects of dark matter and the intrinsic shape of the velocity dispersion profile (see, e.g., Cappellari et al. 2006; Gallazzi et al. 2006; van der Wel et al. 2006). By assuming a constant $k$ for all galaxies, this expression implicitly assumes that all galaxies are dynamically homologous, or self-similar. ${ }^{5}$

But it is important to remember that the observed velocity dispersion is actually the luminosity-weighted mean of the true, radially dependent velocity dispersion, projected onto the line of sight, and within the spectroscopic aperture. The shape of the mass profile has a strong influence on the spatial and dynamical distribution of stellar orbits: in general, the relation between the observed velocity dispersion and the underlying mass profile thus depends on structure as well as size. As a dynamical mass estimator, $\tilde{M}_{\mathrm{d}}$ can therefore only be considered as approximate. (The tilde in $\tilde{M}_{\mathrm{d}}$ is intended to remind the reader of this fact.)

Bertin et al. (2002) provide an analytic expression that makes it possible to approximately account for this effect. Using

\footnotetext{
5 But see also Wolf et al. (2010), who derive a mathematically identical relation from the spherical Jeans equation for a system in dynamical equilibrium.
}

their formulation of the problem, the dynamical mass can be expressed as

$$
\mathrm{G} M_{\mathrm{d}, n}=K_{V}(n) \sigma_{0}^{2} R_{\mathrm{e}} .
$$

Here, the term $K_{V}(n)$ encapsulates the effects of structure on stellar dynamics. (The subscript $n$ in $M_{\mathrm{d}, n}$ is intended to make it clear that non-homology has been accounted for as a function of the Sérsic index, $n$.) For convenience, we will refer to $M_{\mathrm{d}, n}$ as a "structure-corrected" dynamical mass estimator, but we note that the inclusion of a structure-dependent term is not strictly a correction. Bertin et al. (2002) also provide an analytical approximation for $K_{V}(n)$ :

$$
K_{V}(n) \cong \frac{73.32}{10.465+(n-0.95)^{2}}+0.954 .
$$

This expression for $K_{V}(n)$ has been derived assuming a spherical mass distribution that is dynamically isotropic and non-rotating, and which, in projection, follows a Sérsic $(1963,1968)$ surface density profile. For this (admittedly simple) scenario, this approximate expression for $K_{V}(n)$ is accurate at the percent level for $1 \leqslant n \leqslant 10$. Substituting trial values of $n=2$ and $n=8$ into Equation (3) suggests that the differential effect of non-homology on the inferred value of the dynamical mass is as much as a factor of 3 , or 0.5 dex. Our first task in this paper, then, will be to explore the importance of structural differences between galaxies, using this prescription.

Before we begin, note that there are alternative approaches to exploring the consistency between stellar- and dynamical mass estimates. In particular, a number of authors have considered the relation between galaxies' stellar and dynamical masses in the context of well-known scaling relations between luminosity/mass and dynamics. For example, Bell \& de Jong (2001) considered the relation between baryonic (cf. stellar) mass, $M_{\mathrm{bar}}$, and circular rotation velocity, $V_{C}$, for disk galaxies - the baryonic Tully-Fisher relation. These authors showed that stellar mass estimates based on different passbands (i.e., $M_{*} / L_{V}$ versus $\left.M_{*} / L_{K}\right)$ produced consistent $M_{\mathrm{bar}}-V_{c}$ relations. Furthermore, for a fixed IMF, they argued that it was possible use a single color to estimate stellar mass-to-light ratios with an accuracy of $0.1-0.2$ dex.

There have also been a number of analogous studies for elliptical galaxies, based on the fundamental plane (Djorgovsky \& Davis 1987; Dressler et al. 1987), which can be understood as a correlation between the dynamical mass-to-light ratio, $M_{\mathrm{d}} / L$, and surface brightness. These studies (see, e.g., Cappellari et al. 2006, and references therein) have tended to focus on the "tilt" of the fundamental plane - that is, the deviation of the observed relation from the expectation assuming both a constant $M_{*} / L$ and structural and dynamical homology for all early-type galaxies. The tilt of the fundamental plane thus offers a means of probing variations in $M_{\mathrm{d}} / L$ (including both variations in $M_{*} / L$ due to different stellar populations, and variations in $M_{*} / M_{\mathrm{d}}$ due to, e.g., different dark-to-luminous mass ratios) and/or the degree of non-/homology. While the relative importance of these different effects remains an open question, it seems clear that, at least for early-type galaxies, both $M_{*} / L$ and $M_{*} / M_{\mathrm{d}}$ vary systematically with mass (see, e.g., Prugniel \& Simien 1996; Bertin et al. 2002; Trujillo et al. 2004; Cappellari et al. 2006; La Barbera 2008; Allanson et al. 2009).

This paper is structured as follows: in Section 2, we describe the various SDSS-derived catalogs that we will use, including the definition of our galaxy sample. We validate the velocity dispersion measurements used to derive dynamical masses in 
Appendix A. In Sections 3 and 4, we present two parallel comparisons between stellar and dynamical mass estimates for galaxies in our sample. First, in Section 3, we use the simple dynamical mass estimate $\tilde{M}_{\mathrm{d}}$; then, in Section 4 we show how the comparison changes using the structure-corrected dynamical mass estimate, $M_{\mathrm{d}, n}$. In Section 5, we explore the consistency between $M_{\mathrm{d}, n}$ and $M_{*}$. In particular, we will show that there are no statistically significant trends in the ratio $M_{*} / M_{\mathrm{d}, n}$ that would indicate measurement biases in $M_{*}$ and/ or $M_{\mathrm{d}, n}$; this is not the case for the simple dynamical mass estimate $\tilde{M}_{\mathrm{d}}$. We show in Appendix B that these results are not unique to the sample we consider in the main text. We discuss the interpretation and implications of this result in Section 6, before providing a summary of our main results and conclusions in Section 7.

Throughout this work, we will assume the concordance cosmology; viz., $\left(\Omega_{m}, \Omega_{\Lambda}, \Omega_{0}\right)=(0.3,0.7,1.0)$, and $H_{0}=$ $70 \mathrm{~km} \mathrm{~s}^{-1} \mathrm{Mpc}^{-1}$, and adopt a Chabrier (2003) IMF.

\section{DATA}

This work is based on data drawn from several publicly available catalogs based on the SDSS data set. Our analysis is based on redshifts and velocity dispersions from the basic SDSS catalog for DR7 (Abazajian et al. 2009). ${ }^{6}$ We use Sérsic-fit structural parameters from Guo et al. (2009, hereafter G09) and SED-fit stellar mass-to-light ratio measurements from the DR7 Max-Planck-Institute for Astrophysics (MPA)/Johns Hopkins University (JHU) value added catalog. ${ }^{7}$ In Appendix B, we repeat our analysis using the Sérsic-fit structural parameters given in the New York University (NYU) Value Added Galaxy Catalog (VAGC; Blanton et al. 2005b) for DR7. Each of these catalogs have been well described and documented in the references given; in this section, we only briefly summarize the most relevant aspects of each catalog for the present work.

\subsection{Redshifts and Velocity Dispersions}

There are two sets of redshift and velocity dispersion measurements given in the basic SDSS catalog for DR6 and DR7: the "spectro1D" values produced by the Chicago group, and the "specBS" values produced by the Princeton group. In terms of redshifts, the two algorithms produce virtually identical results. The major difference between the two algorithms is that, whereas the Chicago pipeline only gives velocity dispersion measurements to those galaxies that are spectrally classified as being "early type," all galaxies are given a velocity dispersion measurement by the Princeton pipeline. From DR6, both the Princeton and Chicago velocity dispersion measurement algorithms have been updated, so as to eliminate the systematic bias at low dispersions identified by Bernardi (2007) for the DR5 values. ${ }^{8}$ In Appendix A, we compare both sets of velocity dispersions to those given by Faber et al. (1989) for bright, early-type galaxies: in both cases, the values agree with the Faber et al. (1989) catalog values with an rms difference of $\sim 18 \mathrm{~km} \mathrm{~s}^{-1}$ and no discernible systematic bias.

The default redshifts and velocity dispersions in the SDSS catalog (specifically, using SDSS parlance, the parameters $z$

\footnotetext{
6 Accessed via the Catalog Archive Server (CAS; Thakar et al. 2008): http://casjobs.sdss.org/CasJobs/.

7 Available via http://www.mpa-garching.mpg.de/SDSS/.

8 See http://www.sdss.org/dr7/algorithms/veldisp.html for a discussion of the spectro1D and specBS algorithms, as well as a comparison between these values and those from Bernardi et al. (2003a, 2003b) and SDSS DR5.
}

and veldisp given in the table specObjAll) are the Chicago values. For the sole reason that Princeton velocity dispersions are given for all galaxies (rather than only the spectroscopically early types), we have elected to use the Princeton values instead; these are also the measurements adopted for both the NYU and the MPA/JHU value added catalogs.

As we have mentioned in Section 1, the observed velocity dispersion is the luminosity-weighted average within the (projected) spectroscopic aperture. In order to account for aperture effects, we have scaled the observed value, $\sigma_{\mathrm{ob}}$, for each galaxy to a central velocity dispersion, $\sigma_{0}$, which is defined to be that which would be observed within a circular aperture with a radius equal to $1 / 8$ times the apparent effective radius, $\Theta_{\mathrm{e}}$ (see, e.g., Jørgensen et al. 1995). This correction has been made assuming $\sigma(R) \propto R^{-0.066}$; i.e., $\sigma_{0} / \sigma_{\mathrm{ob}}=\left(8 \Theta_{\mathrm{ap}} / \Theta_{\mathrm{e}}\right)^{-0.066}$, where $\Theta_{\mathrm{ap}}=1^{\prime \prime} .5$ is the radius of the SDSS spectroscopic aperture. The scaling of $\sigma(R)$ has been derived by taking a luminosityweighted integral of the spatially resolved velocity dispersions of galaxies from the SAURON survey (Cappellari et al. 2006). The corrections themselves are small-the median correction is 0.02 dex, with an rms scatter of 0.02 dex - and do not have a major impact on our results. Our qualitative conclusions do not change if we assume the slightly weaker radial dependence $\sigma(R) \propto R^{-0.04}$ as found by Jørgensen et al. (1995), or if we neglect this correction altogether.

\subsection{Sérsic Parameters: Size, Flux, and Structure}

G09 have derived $r$-band structural parameters including total magnitude, $m_{\text {tot }}$, effective radius, $\Theta_{\mathrm{e}}$, and Sérsic index, $n$, for a modest sized sample of SDSS galaxies. (We discuss the specific sample selection in Section 2.4.) These values have been derived via parametric fits to the (two-dimensional) $r$-band surface brightness distribution of each galaxy, assuming a Sérsic $(1963,1968)$ profile, and convolved with the appropriate pointspread function (PSF), using the publicly available code galf it (Peng et al. 2002). In order to account for blending, where two galaxies are very close, both the target and companion(s) are fit simultaneously. Through analysis of simulated data, G09 show that the median error in each of $m_{\text {tot }}, \Theta_{\mathrm{e}}$, and $n$ to be less than $10 \%$. (Although at the same time, they show that the uncertainties on the derived parameters associated with background subtraction alone can be significantly greater than this.)

In Appendix B, we will also make use of Sérsic-fit structural parameters from the NYU VAGC (Blanton et al. 2005b). Whereas galfit considers the two-dimensional surface brightness distribution, the VAGC algorithm makes fits to the onedimensional azimuthally averaged curve of growth. The analysis of simulated data presented by Blanton et al. (2005b) shows the VAGC Sérsic parameters to be systematically biased toward low fluxes, sizes, and Sérsic indices. This problem becomes progressively worse for larger $n$, such that sizes are underestimated by $\gtrsim 20 \%$ and fluxes by $\gtrsim 10 \%$ for $n \gtrsim 5$. G09 have shown that this bias is produced by background overestimation and oversubtraction in the VAGC Sérsic fits, owing to the use of a "local," rather than a "global" background estimator.

\subsection{Stellar Masses}

We note that there are rather large differences between the Sérsic magnitudes given by G09 and the default model magnitudes given in the SDSS catalog. The model photometry is derived by making parametric fits to the two-dimensional 
surface brightness distribution in each band, using the sector fitting technique described by Strauss et al. (2002). These fits assume either an exponential or a De Vaucouleurs profile; the profile shape is chosen based on the best-fit $\chi^{2}$ in the $r$ band. For galaxies that are best fit by a De Vaucouleurs model, we find $\left(m_{\mathrm{G} 09, r}-m_{\mathrm{DeV}, r}\right) \approx-0.26+0.11(n-4)$, where $n$ is the Sérsic index reported by G09; the scatter around this relation is at the level of $0.15 \mathrm{mag}(1 \sigma)$. That is, even where G09 find $n=4$, their flux is approximately $0.26 \mathrm{mag}$ brighter than the SDSS (De Vaucouleurs) model flux; this discrepancy is larger for larger values of $n$. For this reason, we take the $r$-band Sérsic magnitude from G09 as a measure of total flux.

To derive a stellar mass, we then use $M_{*} / L$ s taken from the MPA-JHU catalog (DR7), which is maintained by the Garching group. ${ }^{9}$ Note that, unlike previous MPA-JHU catalogs (e.g., Kauffmann et al. 2003a; Brinchmann et al. 2004; Gallazzi et al. 2005), which were based on the SDSS spectroscopy, these masses are derived from fits to the ugriz model SEDs. ${ }^{10}$ Note, however, that the SED photometry has been corrected for emission lines, according to the line-to-continuum flux ratio in the spectroscopic fiber aperture (we discuss the importance of this correction in Section 5.3). The SED fits are based on the synthetic stellar population library described by Gallazzi et al. (2005), which have been constructed using the Bruzual \& Charlot (2003) stellar population models, assuming a Chabrier (2003) stellar IMF. These $M_{*} / L$ s have been shown to be in good agreement (rms in $\Delta \log M_{*}$ of $\lesssim 0.1 \mathrm{dex}$ ) with the spectrally derived values described by Kauffmann et al. (2003a) for DR4. ${ }^{11}$

\subsection{Sample Definition}

Our decision to rely on the Sérsic structural parameters derived by G09 restricts us to working with their sample. Our rationale for this decision is that, in our estimation, the G09 Sérsic fits are the most robust that are presently available.

The G09 sample was constructed with the specific goal of exploring differences in the sizes and structures of "central" and "satellite" galaxies in groups and clusters. To this end, they selected $911 z<0.08$ "centrals" as the first-ranked (in terms of $M_{*}$ ) group/cluster members from the Yang et al. (2007) group catalog, which was in turn constructed from the DR4 NYU VAGC. These galaxies were selected to have a flat logarithmic distribution in halo mass in the range $11.85<\log M_{\text {halo }} / M_{\odot}<$ 13.85 (800 galaxies), plus 100 galaxies in the range $13.85<$ $\log M_{\text {halo }} / M_{\odot}<14.35$, and all 11 central galaxies in clusters with $\log M_{\text {halo }} / M_{\odot}>14.35$. In this way, the central galaxy sample was constructed to span a representative range of (large) halo masses.

G09 also construct two $z<0.08$ "satellite" control samples, in which the satellite galaxies are selected to match the central galaxies. For the first of these, satellites are chosen to match centrals in $M_{*}$ to within $0.08 \mathrm{dex}$; in the second, satellites are also required to match centrals to within 0.03 mag in ${ }^{0.1}(g-i)$ color. Because more massive galaxies are more likely to be (counted as) centrals, not every central has a satellite counterpart within these limits: the matching is more than $90 \%$ successful for $M_{*}<10^{10.85} M_{\odot}$, and less than $10 \%$ successful

\footnotetext{
9 Available via http://www.mpa-garching.mpg.de/SDSS/DR7/.

10 Note that in the SDSS algorithm, when deriving the ugiz model photometry, the structural parameters in the fits are held fixed to the $r$-band values; only the overall normalization (i.e.,total flux) is allowed to vary. The fits in each band are also convolved with the appropriate PSF. In this sense, the model SEDs are both aperture matched, and PSF corrected.

11 See http://www.mpa-garching.mpg.de/SDSS/DR7/mass_comp.html.
}

for $M_{*}>10^{11.15} M_{\odot}$. The two satellite samples, so constructed, consist of 769 and 746 galaxies, respectively.

G09 exclude a number of these galaxies from their analysis because of confusion, leaving a sample of 879 central galaxies, and two samples of 704 and 696 satellites each. While duplicates are not allowed within the individual satellite samples, some galaxies do appear in both samples; combining the two satellite samples we have 1167 unique galaxies. We exclude a further 71 galaxies whose spectra are not deemed "science worthy" by the SDSS team (i.e., the flag sciencePrimary is set to zero). In order to avoid very large errors in the dynamical mass estimates, we also exclude 160 galaxies that have relative errors in their velocity dispersion measurements that are greater than $10 \%$. This requirement excludes mostly low- $n$ and low- $M_{*}$ galaxies: the vast majority of these 160 galaxies have $M_{*}<10^{10.8} M_{\odot}$ and $n<1.5$. Our results do not depend on these selections. We are thus left with a sample of 1816 galaxies, of which 784 have been selected as central galaxies, and 1032 have been selected as being satellites of comparable mass.

The major disadvantage to using the G09 sample is that the relative number of central/satellite galaxies is not at all representative of the general galaxy population. However, it is worth noting that G09 have shown that, at least for structurally early-type galaxies, and after matching both color and mass, there are no differences in the sizes and structures of central and satellite galaxies. They thus conclude that the distinction between central and satellite has no impact on galaxy structure. This already suggests that the G09 sample may be adequately statistically representative of the massive galaxy population.

Even so, we will explicitly examine the possible role of sample selection effects in shaping our results in Section 5.1 by comparing different subsamples from within the combined G09 sample. Further, in Appendix B, we analyze a more general galaxy sample, using structural parameters from either the NYU VAGC Sérsic fits or the SDSS De Vaucouleurs/exponential model fits.

\section{RESULTS I-COMPARING STELLAR AND DYNAMICAL MASS ESTIMATES ASSUMING DYNAMICAL HOMOLOGY}

In this section and the next, we present parallel comparisons between stellar mass and two different estimates of dynamical mass. As we have said in Section 1, it is common practice to obtain a simple dynamical mass estimate based on $\sigma_{0}$ and $R_{\mathrm{e}}$ alone, using the scalar virial theorem, viz., $\tilde{M}_{\mathrm{d}} \approx k \sigma_{0}^{2} R_{\mathrm{e}}$. In Section 3.1 , we directly compare the values of $M_{*}$ and $\tilde{M}_{\mathrm{d}}$ for the G09 galaxies; we will assume $k=4$. We will then argue in Section 3.2 that the agreement between stellar and dynamical mass estimates may be significantly improved if we allow for non-homology. To test this idea, in Section 4, we will perform the same comparisons using the structure-corrected dynamical mass estimator, $M_{\mathrm{d}, n}$.

\subsection{The Relation Between Stellar and Dynamical Mass}

In Figure 1, we compare the values of the simple dynamical mass estimator, $\tilde{M}_{\mathrm{d}}$, to the values of $M_{*}$ for galaxies in the G09 sample. The first thing to notice is that there is a relatively tight but clearly nonlinear relation between $\tilde{M}_{\mathrm{d}}$ and $M_{*}$, such that $M_{*} \propto \tilde{M}_{\mathrm{d}}^{a}$ with $a<1$. Moreover, this simple analysis suggests that for many galaxies, including the majority of galaxies with $\tilde{M}_{\mathrm{d}} \lesssim 10^{10.5} M_{\odot}, M_{*}$ actually exceeds $\tilde{M}_{\mathrm{d}}$. This is 
logically inconsistent, and necessarily implies a problem in the calculation of $M_{*}$ and/or $\tilde{M}_{\mathrm{d}}$.

Before discussing this result further, it is appropriate to make a few comments about the random errors in our estimates of $M_{*}$ and $\tilde{M}_{\mathrm{d}}$. In particular, it is important to realize that the errors in $\Theta_{\mathrm{e}}, m_{\mathrm{tot}}$, and $n$ are strongly covariant: for example, an error in the structural index will affect the values of both $\Theta_{\mathrm{e}}$ and $m_{\text {tot }}$. Because $M_{*}$ depends on the measured value of $m_{\text {tot }}$, and $\tilde{M}_{\mathrm{d}}$ on the measured value of $\Theta_{\mathrm{e}}, M_{*}$ and $\tilde{M}_{\mathrm{d}}$ are thus also covariant. This makes the seemingly trivial task of fitting a line to the observed $M_{*}-\tilde{M}_{\mathrm{d}}$ relation rather problematic. To do this properly would require full and consistent treatment of the covariant uncertainties in the Sérsic-fit parameters, but this information is not given by G09.

Our solution to this problem is simply to minimize the mean absolute perpendicular distance between the data and the fit. When doing so, we also use a "sigma-clipping" algorithm to avoid the influence of the most egregious outliers; specifically, we iteratively exclude points that lie off the best-fit line by more than five times the rms offset. While the gradient of the best-fit line does depend on the fitting scheme used (we will explore this in more detail in Section 4.1), the best-fit parameters are not strongly dependent on how aggressively we sigma-clip. In order to avoid strong covariances between the slope and intercept of the best-fit line, we actually compute the fit in terms of $\log \left(\tilde{M}_{\mathrm{d}} / 10^{11} M_{\odot}\right)$; that is, we fit a relation of the form $y=a(x-11)+b_{11}$. Statistical uncertainties on the fit parameters have been derived from bootstrap resampling. The best fit to the $M_{*}-\tilde{M}_{\mathrm{d}}$ relation, so derived, is shown as line heavy dashed line in Figure 1. The best-fit parameters are $a=0.73 \pm 0.007$ and $b_{11}=-0.14 \pm 0.003$.

In Figure 2, we explore the relation between $M_{*}$ and $\tilde{M}_{\mathrm{d}}$ in greater detail. The different panels of this figure show the difference between $M_{*}$ and $\tilde{M}_{\mathrm{d}}$ as a function of several interesting global properties: (from left to right) galaxy mass, structure, dynamics, and surface density. It is clear that $M_{*} / \tilde{M}_{\mathrm{d}}$ is strongly correlated with all four of these parameters. For each of the parameters shown, the size of the median trend in $M_{*} / \tilde{M}_{\mathrm{d}}$ across the sample is on the order of $0.5 \mathrm{dex}$, although it is slightly lower for $M_{*}$ and slightly higher for effective surface density.

To quantify this statement, we have again made fits to the data, assuming the form $y=a(x-X)+b_{X}$, where $X$ is an arbitrary value chosen to be close to the median value of the quantity $x$ for our sample. For these fits, in contrast to the previous section, we have minimized the mean absolute vertical offset between the data and the fit. Again, we use a non-aggressive sigma-clipping scheme to exclude extreme outliers. (In all that follows, when considering the stellar-to-dynamical mass ratio, we will always fit in this way; we will only use the minimum perpendicular distance algorithm described above when fitting the relation between stellar and dynamical masses.) The best-fit lines to the data, so derived, are shown as the heavy dashed lines in each panel; the best-fit parameters are given in each panel. In the case of Sérsic index, the scatter around the best-fit relation is $\approx 0.12 \mathrm{dex}$; for the other three parameters it is slightly higher: $\approx 0.15$ dex.

\subsection{The Importance of Galaxy Structure in Dynamical Mass Estimates}

There are of course strong correlations between mass, velocity dispersion, surface density, and structure. It is thus possible that the apparent trend with any given parameter in Figure 2

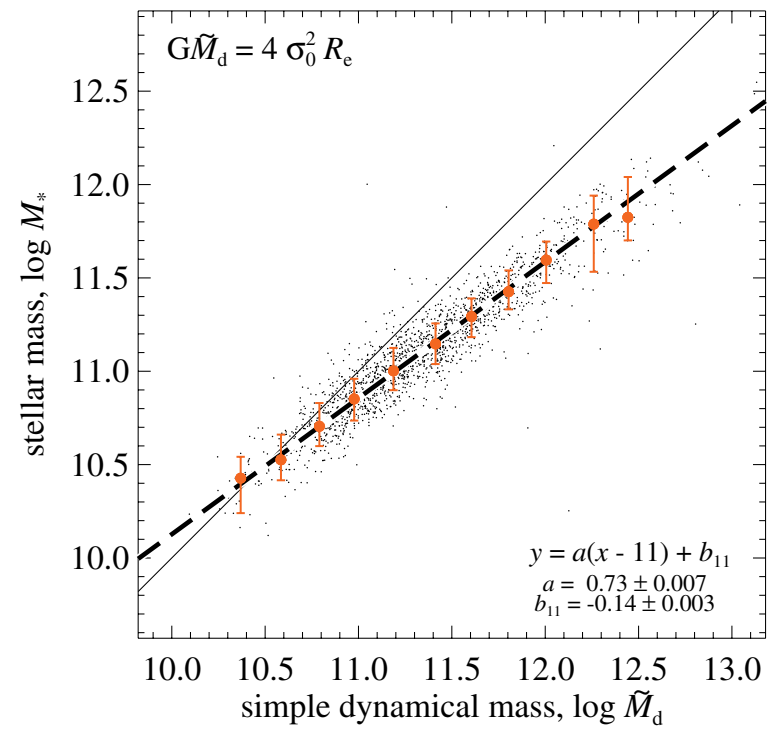

Figure 1. Comparing stellar- and dynamical mass estimates under the assumption of dynamical homology. This figure shows the relation between stellar mass and a simple estimate of dynamical mass, $G \tilde{M}_{\mathrm{d}}=4 \sigma_{0}^{2} R_{\mathrm{e}}$, for galaxies in the G09 sample. The black points show the data themselves; the red points with error bars show the median and $16 / 84$ percentile values of $M_{*}$ in narrow bins of $\tilde{M}_{\mathrm{d}}$; and the heavy dashed line shows a log-linear fit to the data, with the form and parameters as given. While there is a relatively tight correspondence between $M_{*}$ and $\tilde{M}_{\mathrm{d}}$, the relation is clearly nonlinear. Further, for $\tilde{M}_{\mathrm{d}} \lesssim 10^{10.5} M_{\odot}, M_{*}$ appears to exceed $\tilde{M}_{\mathrm{d}}$ for the majority of galaxies, which is logically inconsistent. We explore these results in greater detail in Figures 2 and 3. In Figure 4, we show how these results change if we account for structural and dynamical non-homology in our estimates of dynamical mass.

(A color version of this figure is available in the online journal.)

is "spurious," in the sense that it is driven by a trend in another more "fundamental" parameter. We note that galaxies' star formation activity and histories have been shown to correlate closely with all of mass, velocity dispersion, and surface density (see, e.g., Kauffmann et al. 2003a, 2003b, 2006; Franx et al. 2008; Graves et al. 2009). Indeed, with the assumption that $M_{*} \sim M_{\mathrm{d}, n}$, these three quantities are all related by factors of $R_{\mathrm{e}}$, which is also closely correlated with $M_{*}$ (Shen et al. 2003; Franx et al. 2008). But the fact that $M_{*} / \tilde{M}_{\mathrm{d}}$ depends on galaxy structure - and particularly the agreement between the observed trend and expectations derived from a simple dynamical model-immediately suggests that structure-dependent differences in galaxy dynamics may play a role in the results shown in Figure 1. With this in mind, in Figure 3 we attempt to separate out the $M_{*}$ - and $n$-dependences of $M_{*} / \tilde{M}_{\mathrm{d}}$. Specifically, we want to test the hypothesis that departure from linearity in the $M_{*}-\tilde{M}_{\mathrm{d}}$ relation seen in Figure 1 is at least in part a function of structure, and not mass.

Figure 3(a) shows $M_{*} / \tilde{M}_{\mathrm{d}}$ as a function of $M_{*}$; the colored lines show the median relation in bins of Sérsic index. The median relation between $M_{*} / \tilde{M}_{\mathrm{d}}$ and $M_{*}$ has a rather similar slope for each different $n$-bin: $M_{*} / \tilde{M}_{\mathrm{d}}$ does depend on mass. If the dynamical mass-to-light ratio were to depend on mass only, however, we would expect the relations for different Sérsic indices to overlap. Instead, the relations for each bin are clearly offset from one another. That is, at fixed mass, the scatter in $M_{*} / \tilde{M}_{\mathrm{d}}$ is closely correlated with galaxy structure.

In Figure 3(b), we do the opposite: in this panel, we plot $M_{*} / \tilde{M}_{\mathrm{d}}$ as a function of Sérsic index, and the different lines show the median relation in bins of stellar mass. Again, it is clear that $M_{*} / \tilde{M}_{\mathrm{d}}$ depends on both $M_{*}$ and $n$ : the median relations 


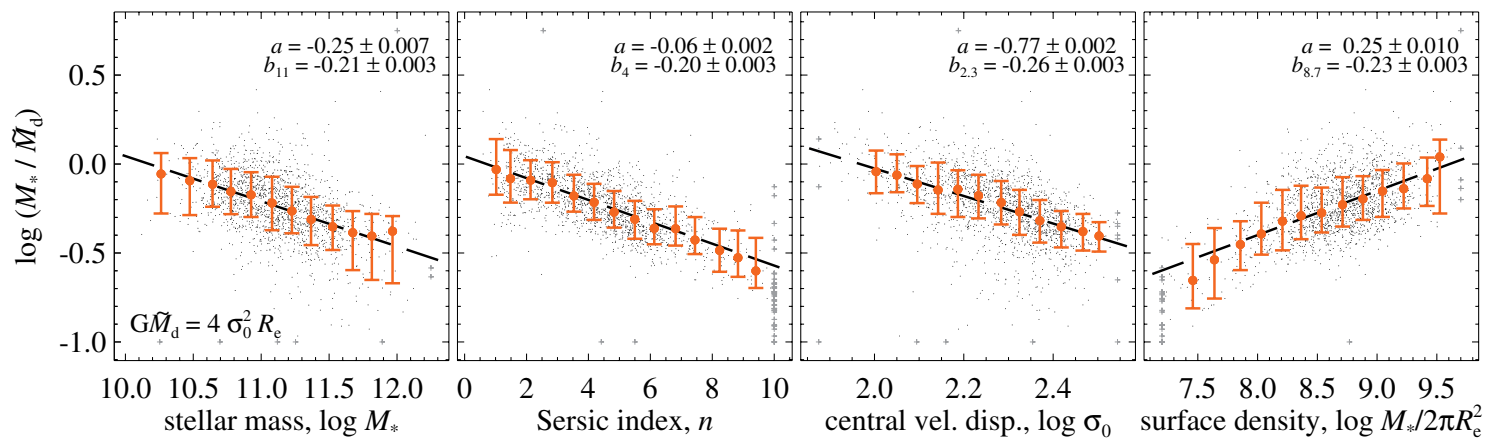

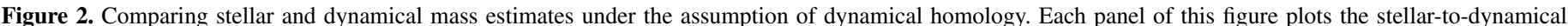

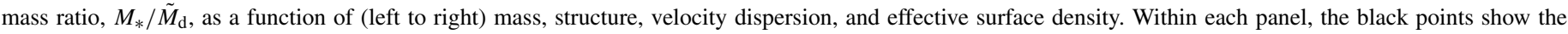

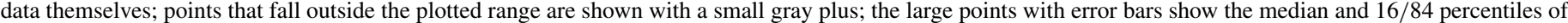

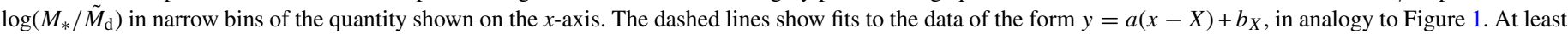

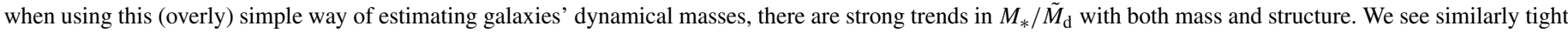
and strong trends with velocity dispersion and effective surface density, as well as with other parameters like size (not shown).

(A color version of this figure is available in the online journal.)
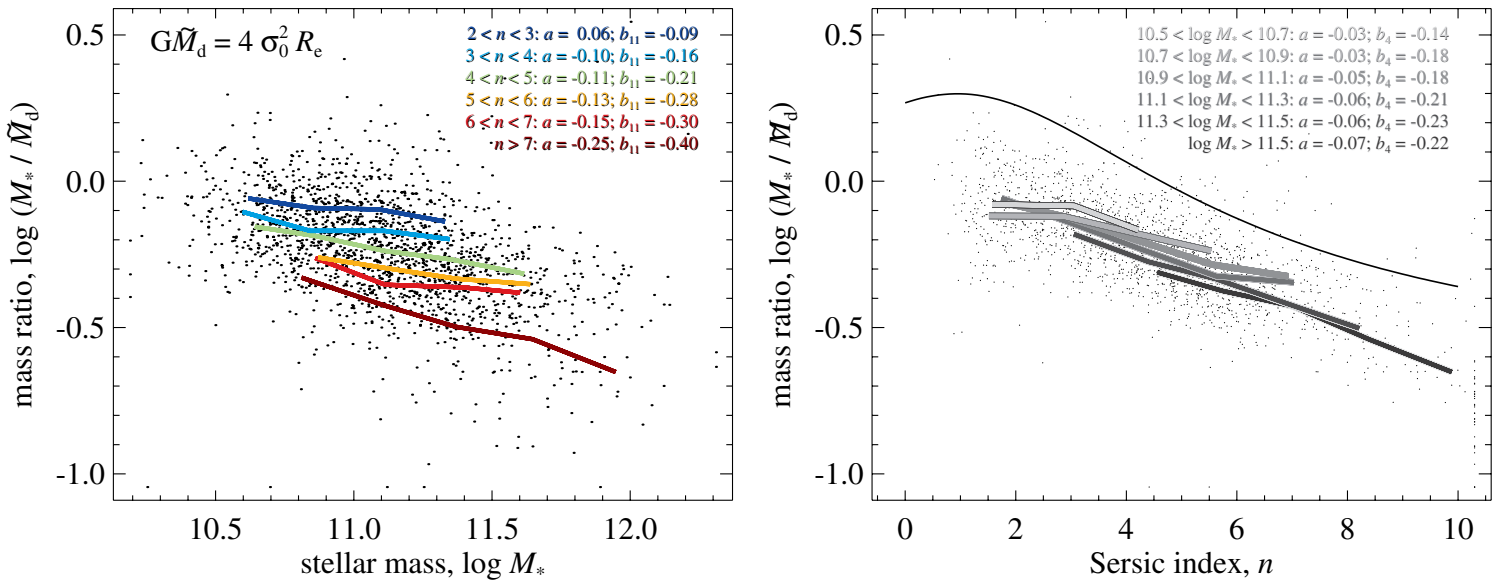

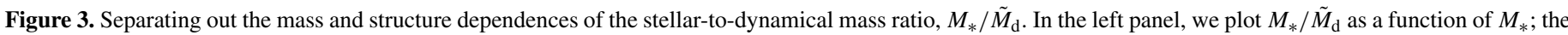

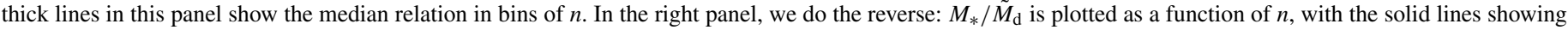

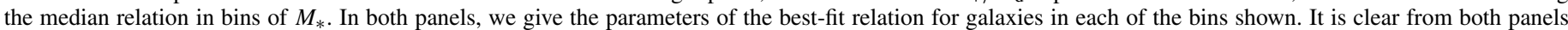

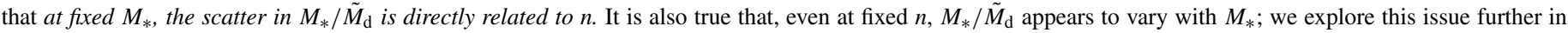

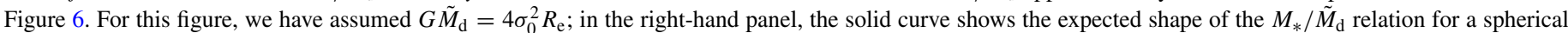

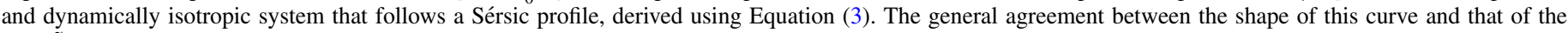

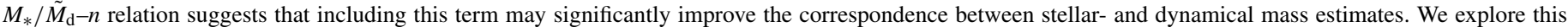
issue further in Figures 4 and 5.

(A color version of this figure is available in the online journal.)

for each different mass bin are roughly parallel, but offset from one another.

Further, the different mass bins in Figure 3(b) cover different ranges in Sérsic index: where the lowest mass bin is dominated by galaxies with $1<n<4$, the highest mass bin is dominated by $n>4$ galaxies. Similar behavior can be seen in Figure 3(a): the lowest $n$-bin contains very few galaxies with $\log M_{*}>$ $11.3 M_{\odot}$, and virtually all $\log M_{*}>11.5 M_{\odot}$ galaxies are in the $n>7 \mathrm{bin}$. That is, there is a correlation between $M_{*}$ and $n$ within our sample. Because the trend is toward lower values of $M_{*} / \tilde{M}_{\mathrm{d}}$ with increasing $n$, this correlation contributes to the apparent mass dependence of the dynamical-to-stellar mass ratio.

Again, the dynamical mass estimates used thus far have been derived under the assumption of dynamical homology (i.e., $K_{V}$ is equal to 4 for all galaxies). The solid line in Figure 3(b) shows the expected shape of the relation between $M_{*} / \tilde{M}_{\mathrm{d}}$ and $n$, derived from Equation (3). The agreement between the observed relation between $M_{*} / \tilde{M}_{\mathrm{d}}$ and $n$ and the expectations from this (admittedly simple) dynamical model immediately suggests that non-homology has an important effect on dynamical mass estimates. We note that both Prugniel \& Simien (1996) and Trujillo et al. (2004) have made a similar argument for the importance of non-homology based on the fundamental plane of elliptical galaxies (see also Section 6).

\section{RESULTS II-COMPARING STELLAR AND DYNAMICAL MASS ESTIMATES ACCOUNTING FOR DYNAMICAL NON-HOMOLOGY}

In this section, we investigate the potential impact of nonhomology on the agreement between stellar and dynamical mass estimates. To this end, we repeat the comparisons between stellar and dynamical mass estimates presented above, using the structure-corrected dynamical mass estimator, $M_{\mathrm{d}, n}$, in place of the simple estimate $\tilde{M}_{\mathrm{d}}$. We quantify the relation between $M_{*}$ and $M_{\mathrm{d}, n}$ for our sample in Section 4.1, and show in Section 4.2 that allowing for non-homology considerably improves the correspondence between stellar and dynamical mass estimates. 




Figure 4. Comparing stellar- and dynamical mass estimates, accounting for both structural and dynamical non-homology. The difference between this figure and Figure 1 is that we have used $G M_{\mathrm{d}, n}=K_{V}(n) \sigma_{0}^{2} R_{\mathrm{e}}^{2}$, with $K_{V}(n)$ defined as in Equation (3); otherwise all symbols and their meanings are as in Figure 1. The key point to be made from this figure, in comparison to Figure 1, is that allowing for non-homology makes a big difference to the inferred dynamical masses, and so to the correspondence between stellar and dynamical masses. Further, we note that the apparent inconsistency whereby $M_{*}>\tilde{M}_{\mathrm{d}}$ for $M_{*} \lesssim 10^{10.5} M_{\odot}$ galaxies seen in Figure 1 is no longer apparent. After accounting for structuredependent differences in galaxies' dynamics, the relation between $M_{*}$ and $M_{\mathrm{d}, n}$ is much more nearly linear. However, it remains true that the difference between stellar and dynamical mass appears to grow with increasing mass.

(A color version of this figure is available in the online journal.)

\subsection{The Relation Between Stellar and Dynamical Mass}

In Figure 4, we show the relation between stellar and dynamical mass for the G09 sample, using the structurecorrected dynamical mass estimator, $M_{\mathrm{d}, n}$; this figure should be compared to Figure 1 . It is immediately obvious that the correlation between $M_{*}$ and $M_{\mathrm{d}, n}$ is much closer to linear than that between $M_{*}$ and the simple dynamical mass, $\tilde{M}_{\mathrm{d}}$. Further, we note that the results are now logically consistent, in that $M_{*}<M_{\mathrm{d}, n}$ for almost all galaxies. This is our most basic result: structure-dependent differences in galaxy dynamics can have a big impact on the inferred dynamical mass, and so the stellar-to-dynamical mass ratio.

The best-fit parameters for the $M_{*}-M_{\mathrm{d}, n}$ relation are $a=$ $0.92 \pm 0.007$ and $b_{11}=-0.23 \pm 0.004$, where we have used bootstrap resampling to estimate the statistical uncertainty. While the statistical errors in the fit parameters are impressively small, systematic errors are sure to dominate. To see this, consider what would happen if we were to impose a stellar mass limit $M_{*}>M_{\text {lim }}$ in Figure 4: we would only include those galaxies with $M_{\mathrm{d}, n}<M_{\lim }$ that have high values of $M_{*} / M_{\mathrm{d}, n}$; similarly, we would exclude those galaxies with $M_{\mathrm{d}, n}<M_{\text {lim }}$ that have low values of $M_{*} / M_{\mathrm{d}, n}$. This would lead to a significantly shallower best-fit slope to the $M_{*} / M_{\mathrm{d}, n}$ relation. As a specific example, if we were only to consider galaxies from the G09 sample with $M_{*}>10^{10.8} M_{\odot}$, we would find $M_{*} \propto M_{\mathrm{d}, n}^{0.86}$. In this context, it is worth noting both that the scatter in the $M_{*}-M_{\mathrm{d}, n}$ relation appears to increase with decreasing mass, and that the lowest mass points in Figure 4 lie systematically above the best-fit line.

Note that, while we have phrased this in terms of a mass selection effect, at least part of this effect is related to how we have fit for the slope of the $M_{*}-M_{\mathrm{d}, n}$ relation; viz., by minimizing the mean perpendicular distance between the data and the best-fit line. If we were instead to fit by minimizing the mean offset in $M_{*} / M_{\mathrm{d}, n}$ as a function of $M_{*}$, we would reduce our vulnerability to this effect.

There are thus two effects that have the potential to systematically bias the measured slope of the $M_{*}-M_{\mathrm{d}, n}$ relation. We can obtain a simple estimate for the systematic error on the parameters $a$ and $b_{11}$ by re-fitting the $M_{*}-M_{\mathrm{d}, n}$ relations in different ways. If we fit by minimizing the mean vertical offset, $\Delta M_{*}$, from the best-fit $M_{*} / M_{\mathrm{d}, n}$ relation, we find $a=0.85$ and $b_{11}=-0.20$. If instead we fit by minimizing the mean horizontal offset, $\Delta M_{\mathrm{d}, n}$, we find $a=1.00$ and $b_{11}=-0.26$. That is, the systematic uncertainties related to the technique used to fit the $M_{*}-M_{\mathrm{d}, n}$ relation (for this sample) are on the order $\Delta a=0.08$ and $\Delta b_{11}=0.03$.

What about the systematic biases due to the particular mass distribution of galaxies in the G09 sample? To explore the importance of these effects in our measurement of the slope of the $M_{*}-M_{\mathrm{d}, n}$ relation, we have tried re-fitting the $M_{*}-M_{\mathrm{d}, n}$ relation, weighting each point according to its stellar mass. The specific weights have been derived through a comparison between the mass distribution of galaxies within the G09 sample, in bins of $\Delta M_{*}=0.1 \mathrm{dex}$, and the $z \sim 0$ mass function of Bell et al. (2003). We have chosen the weight for each galaxy so that that the weighted stellar mass distribution of the G09 sample matches the "real" stellar mass function. This weighting scheme is akin to $1 / V_{\max }$ weighting, inasmuch as if one were able to derive $V_{\max }$ values for the sample, one would hope to obtain similar values.

Re-fitting the G09 sample using these weights, we find $a=1.00 \pm 0.05$. The larger random error on this value in comparison to our fiducial values stems from the fact that the lower mass galaxies are given much greater weights (by several orders of magnitude); the inclusion/exclusion of these points in the bootstrap resampling thus has a major impact on the bestfit slope. The fact that using these weightings gives an almost perfectly linear relation between $M_{*}$ and $M_{\mathrm{d}, n}$ is striking, but it is important to remember that this fit is based almost entirely on the relatively small number of $M_{*} \lesssim 10^{11} M_{\odot}$ galaxies in the sample. In Appendix B, we perform the same analysis for a more general galaxy sample, drawn from the NYU VAGC, which provides a much better sampling of the true galaxy mass function. Using the weighting scheme described above, the relative weights of galaxies with $10.2<\log M_{*} / M_{\odot}<11.7$ differ by a factor of only 10 . For this galaxy sample, we find $a=0.930 \pm 0.004\left({ }_{-0.07}^{+0.03}\right)$ without weighting, compared to $a=0.933 \pm 0.007$ with weighting.

While the data are consistent with a linear relation between $M_{*}$ and $M_{\mathrm{d}, n}$, they thus prefer a slightly shallower relation. For the G09 sample, we find $a=0.93 \pm 0.007( \pm 0.07)$, and $b_{11}=$ $-0.23 \pm 0.004( \pm 0.03)$. This should be compared to the values of $a=0.73 \pm 0.006\left({ }_{-0.03}^{+0.07}\right)$ and $b_{11}=-0.14 \pm 0.003\left({ }_{-0.03}^{+0.01}\right)$ for the simple dynamical mass estimate, $\tilde{M}_{\mathrm{d}}$. While the mass distribution of galaxies within the G09 sample can in principle induce a large bias in the measured slope of the $M_{*}-M_{\mathrm{d}, n}$ relation, our best-fit value is in fact consistent with that derived from a more general galaxy sample, in which these effects play a far smaller role. We will explore the potential role of other sample selection effects in Section 5.2.

In Figure 5, we show the trends in $M_{*} / M_{\mathrm{d}, n}$ with mass, structure, dynamics, and density; this figure should be compared to Figure 2. For each of these four parameters, the trends in 


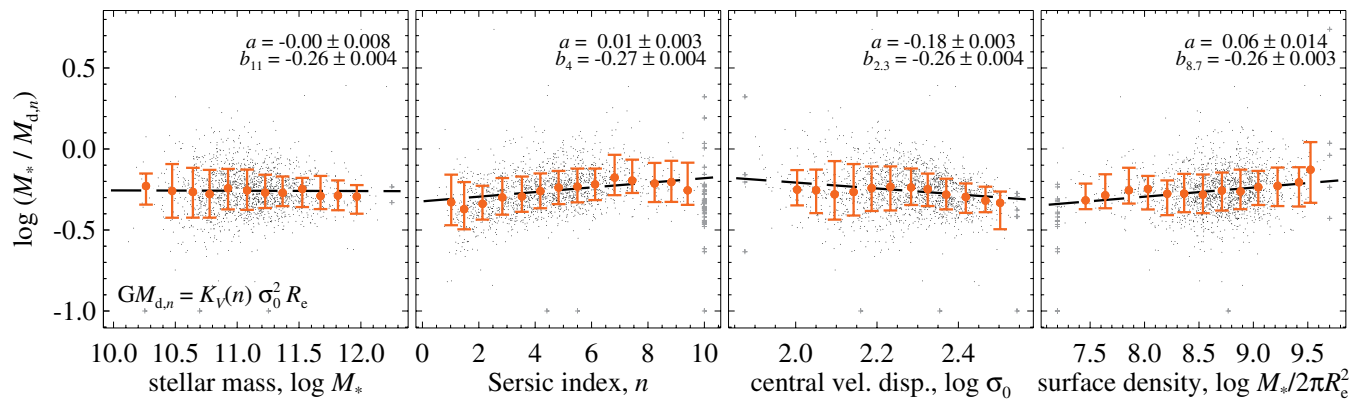

Figure 5. Comparing stellar and dynamical mass estimates accounting for structure-dependent differences in galaxy dynamics. The difference between this figure and Figure 2 is that we have used $G M_{\mathrm{d}, n}=K_{V}(n) \sigma_{0}^{2} R_{\mathrm{e}}^{2}$, with $K_{V}(n)$ defined as in Equation (3); otherwise all symbols and their meanings as is in Figure 1. After accounting for structure-dependent differences in galaxy dynamics, the apparent trends in $M_{*} / M_{\mathrm{d}, n}$ with stellar mass and Sérsic index are substantially reduced. The apparent trends with other properties, including velocity dispersion, surface density, size, and color, are also substantially reduced, or effectively disappear (see also Figures 7 and 9).

(A color version of this figure is available in the online journal.)
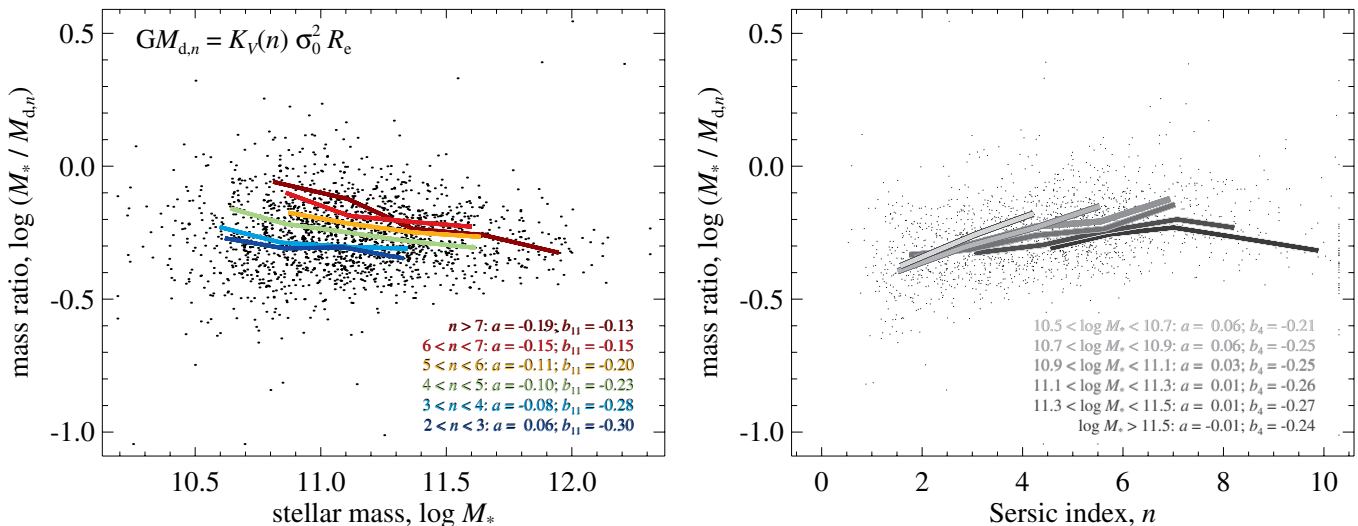

Figure 6. Does $M_{*} / M_{\mathrm{d}, n}$ vary with $M_{*}, n$, or both? The difference between this figure and Figure 3 is that we have accounted for structure-dependent differences in galaxy dynamics through the $K_{V}(n)$ term defined in Equation (3); otherwise all symbols and their meanings as is in Figure 3. As in Figure 3, it is clear that the scatter in $M_{*} / M_{\mathrm{d}, n}$ at fixed $M_{*}$ depends strongly on $n$; conversely, at fixed $n$, there is also a strong correlation between $M_{*}$ and $M_{*} / M_{\mathrm{d}, n}$. This effect appears to be stronger for higher values of $n$ and $M_{*}$. In comparison to Figure 3, the inclusion of an $n$-dependent dynamical term obviously changes the slope of the $M_{*} / M_{\mathrm{d}, n}-n$ relation, but cannot affect the slope of the $M_{*} / M_{\mathrm{d}, n}-M_{*}$ relation at fixed $n$. In other words, the apparent $n$-dependence of $M_{*} / M_{\mathrm{d}, n}$ at fixed $M_{*}$ is sensitive to the specific model used to derive $K_{V}(n)$; on the other hand, the result that, at fixed $n, M_{*} / M_{\mathrm{d}, n}$ varies with $M_{*}$ is insensitive assumed form of $K_{V}(n)$.

(A color version of this figure is available in the online journal.)

$M_{*} / M_{\mathrm{d}, n}$ are significantly weaker than what we have seen for $M_{*} / \tilde{M}_{\mathrm{d}}$. The net differential trend across the sample is now on the order of 0.2 dex or so, as compared to $0.5 \mathrm{dex}$ for $M_{*} / \tilde{M}_{\mathrm{d}}$. While there is still a strongly statistically significant trend in $M_{*} / M_{\mathrm{d}, n}$ with $\sigma$, the trend with surface density is now only significant at the $4 \sigma$ level. While we do still see signs of a trend in $M_{*} / M_{\mathrm{d}, n}$ with $n$, this trend is not statistically significant, at least for the sample as a whole. We discuss this point further in the next section.

\subsection{Does $M_{*} / M_{\mathrm{d}, n}$ Depend on Mass, or Structure, or Both?}

In Figure 6, we return to the issue of the $n$ - and $M_{*^{-}}$ dependence of $M_{*} / M_{\mathrm{d}, n}$; this figure should be compared to Figure 3. In Figure 6(a), we show the median relation between $M_{*} / M_{\mathrm{d}, n}$ and $M_{*}$ in bins of $n$. Again, the trends in $M_{*} / M_{\mathrm{d}, n}$ with $M_{*}$ for the different $n$-bins are parallel, but offset from one another. In each of the $3 \lesssim n \lesssim 7$ bins, we find that $M_{*} / M_{\mathrm{d}, n}$ scales approximately as $\widetilde{M}_{*}^{0.1}$; that is, roughly consistent with the scaling that we see for the sample as a whole. Figure 6(b) shows the median relation between $M_{*} / M_{\mathrm{d}, n}$ as a function of $n$ for different bins in $M_{*}$. While the trend in $M_{*} / M_{\mathrm{d}, n}$ with $n$ is substantially weaker than what we saw using the simple dynamical mass, $\tilde{M}_{\mathrm{d}}$, we still see that $M_{*} / M_{\mathrm{d}, n}$ varies with $n$; if anything, it would appear that by using the prescription for $K_{V}(n)$ given in Equation (3), we have overcorrected for the effects of non-homology. Without detailed dynamical modeling, however, we have no means of refining the model used to derive Equation (3). (We will discuss this point further in Section 6.)

In other words, we have shown that accounting for structural and dynamical homology significantly improves the agreement between stellar and dynamical mass estimates as a function of the Sérsic index, $n$, but we have not unambiguously shown whether or not $M_{*} / M_{\mathrm{d}, n}$ depends on galaxy structure—nor can we.

\section{RESULTS III-EXPLORING POTENTIAL BIASES IN $M_{*} / M_{\mathrm{d}, n}$}

In this section, we discuss three general classes of biases that may affect the results we have presented in Section 4: first, systematic biases in the Sérsic fits that we use to derive $M_{*}$ and $M_{\mathrm{d}, n}$ (Section 5.1; see also Appendix B); then, the possibility of severe selection effects for the G09 sample (Section 5.2; see also Appendix B); and finally, systematic effects associated with the estimation of stellar mass-to-light ratios (Section 5.3). We will show very good consistency between the values of $M_{*}$ and $M_{\mathrm{d}, n}$; this is not the case for the simple estimate $\tilde{M}_{\mathrm{d}}$. As in the previous section, this consistency provides strong circumstantial 


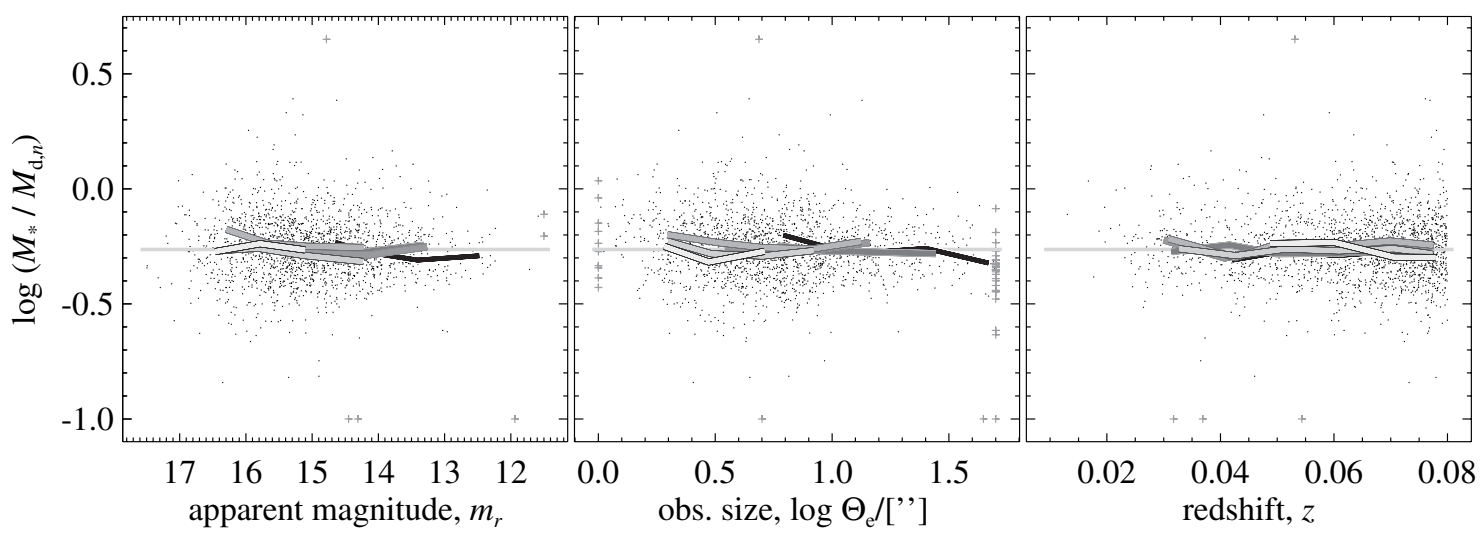

Figure 7. Signs of observational biases? Each panel shows $M_{*} / M_{\mathrm{d}, n}$ a function of a direct observable. Within each panel, the solid lines show the median relation in bins of stellar mass; these bins are the same as those shown in the right-hand panels of Figures 3 and 6 . The points show the data themselves; points that fall outside the range of each panel are shown as a small gray plus. Within the G09 sample, there are correlations between the Sérsic index and each of the observed quantities shown in this figure; however, there are only very weak trends in $M_{*} / M_{\mathrm{d}, n}$ with any of these observables. Moreover, each of the stellar mass bins follows essentially the same median relation. This suggests that neither the stellar nor dynamical mass estimates are obviously seriously biased by systematic errors in the Sérsic fits.

evidence—-but not proof beyond a reasonable doubt—-that there are no significant biases in either measurement.

\subsection{Looking for Possible Observational Biases}

We explore the possibility of serious observational biases in Figure 7. In each panel of this figure, we plot $M_{*} / M_{\mathrm{d}, n}$ as a function of a basic observable: namely (left to right), apparent magnitude, apparent size, and redshift. The lines in each panel show the median relation for the same bins of stellar mass as are shown in Figures 3(b) and 6(b).

By a similar argument to the one given in Section 4.2, if the apparent mass dependence of the ratio $M_{*} / M_{\mathrm{d}, n}$ were driven by observational effects that are strong functions of apparent brightness or size, then we would expect there to be clear trends in $M_{*} / M_{\mathrm{d}, n}$ for each of the stellar mass bins in Figure 7. This is not obviously the case. Fitting to the data in each stellar mass bin, the gradients of the best-fit relation between $M_{*} / M_{\mathrm{d}, n}$ and all of apparent magnitude, apparent size, and redshift are consistent with zero; this is true for each stellar mass bin individually (typically within $1 \sigma$ ), as well as for the sample as a whole

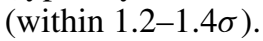

We note that the same is not true using, for example, the Sérsic structural parameters given in the NYU VAGC to derive $M_{*}$ and $M_{\mathrm{d}, n}$ (as we do in Appendix B). In this case, we do see a weakly statistically significant gradient (at the level of $4 \sigma$ ) with observed size. That is, this kind of test is indeed able to (weakly) detect mild systematic errors in the Sérsic-fit parameters on the order of $10 \%-20 \%$. The results in Figure 7 thus argue against the idea that there are any serious biases affecting the measurement of $M_{*}$ or $M_{\mathrm{d}, n}$ (or, more accurately, the ratio $\left.M_{*} / M_{\mathrm{d}, n}\right)$ associated with the Sérsic-fit structural parameters used to derive these values.

As can be seen in Figure 5, there is a statistically significant correlation between $M_{*} / M_{\mathrm{d}, n}$ and velocity dispersion, $\sigma$. Using the same argument as above, it is conceivable that this could be produced by a systematic bias in the measured values of $\sigma$. The observed trend of $\sim-0.12 \mathrm{dex}$ in $M_{*} / M_{\mathrm{d}, n}$ over $\sim 0.5 \mathrm{dex}$ in $\sigma$ could be entirely explained by a $\sim 0.06$ dex differential bias in the measured values of $\sigma$. In connection with this point, we stress that a comparison between the SDSS measured values of $\sigma$ and those of Faber et al. (1989) shows no systematic biases. This should give some confidence that the trend in
$M_{*} / M_{\mathrm{d}, n}$ with $\sigma$ is real, and not a product of observational biases.

\subsection{Looking for Potential Sample Selection Effects}

As we have described in Section 2.4, the G09 sample has been constructed in such a way that massive galaxies in general, and in particular central galaxies in very massive halos, are drastically overrepresented in comparison to the general field population. If there are systematic differences in $M_{*} / M_{\mathrm{d}, n}$ as a function of, for example, environment or star formation activity, there is thus a very real danger that sample selection effects may play an important role in shaping our results.

We explore this issue in Figure 8, in which we have divided the G09 sample in central/satellite and non-/emission subsamples, in order to look for differences between these populations. Here, we have selected "non-emission" galaxies as $\mathrm{H} \alpha$ and $\mathrm{H} \beta$ in absorption. The "emission" subsample contains both starforming galaxies and AGN hosts.

In the upper panels of Figure 8, it is clear that we find essentially identical relations between $M_{*}$ and $M_{\mathrm{d}, n}$ for the central and satellite galaxy subsamples. Given that, as we have shown in Figure $6, M_{*} / M_{\mathrm{d}, n}$ varies with both $M_{*}$ and $n$, it is not all that surprising that central and satellite galaxies show the same $M_{*}-M_{\mathrm{d}, n}$ relation: not only have the two subsamples been constructed to be matched in $M_{*}$, G09 have shown that there are no structural differences between satellites and centrals at fixed mass.

However, we do find very slightly different $M_{*}-M_{\mathrm{d}, n}$ relations for the emission and non-emission subsamples. Formally, the two relations are slightly offset from one another, at the level of $0.05 \mathrm{dex}$; the gradients of the two relations differ at the $2 \sigma$ level. That said, for the $10.5<\log M_{*}<12$ range spanned by the sample, the difference between the best-fit relations for each subsample and that for the sample as a whole are $\lesssim 0.03$ dex. We also note that the apparent offset disappears (at least within $2 \sigma$ ) if we consider only the $n>2$ galaxies in both subsamples. Moreover, we do not find any differences in $M_{*} / M_{\mathrm{d}, n}$ at fixed $M_{*}$ and $n$ between these different subsamples-within statistical uncertainties, each subsample shows the same behavior as is seen in Figure 6. This suggests that the apparent offset between the emission and non-emission galaxies is driven by the different distributions of Sérsic indices within the 

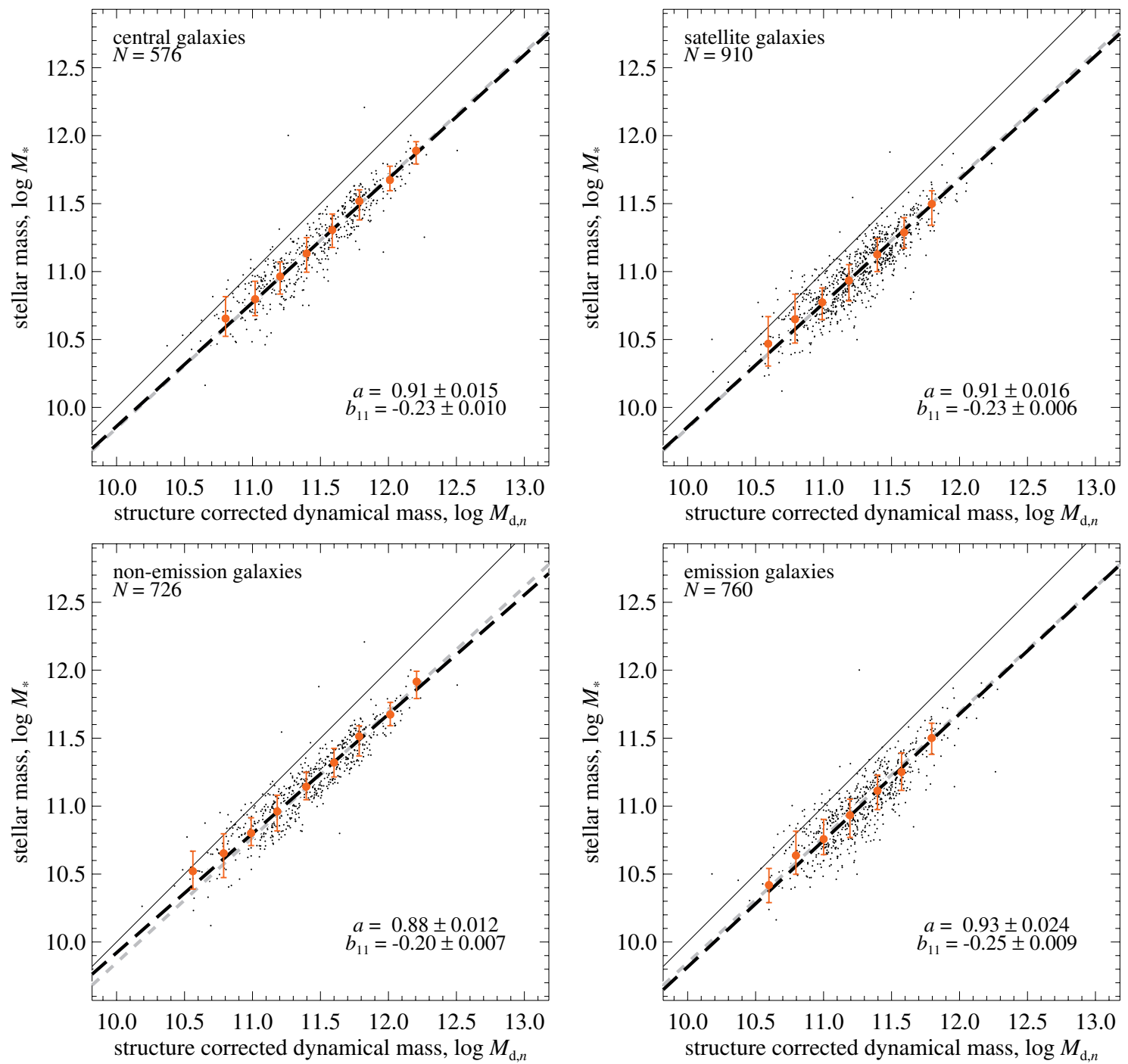

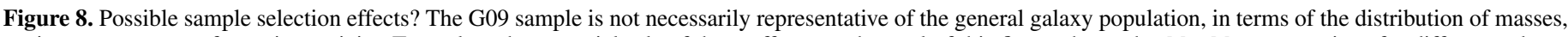

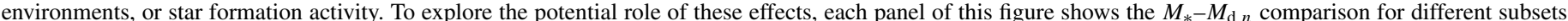

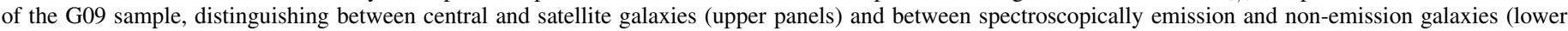

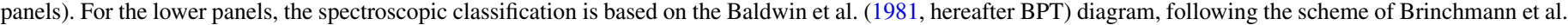

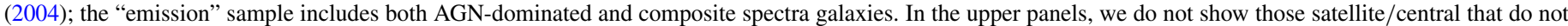

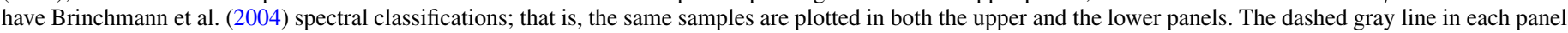

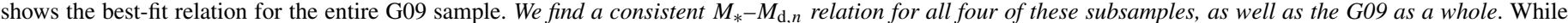

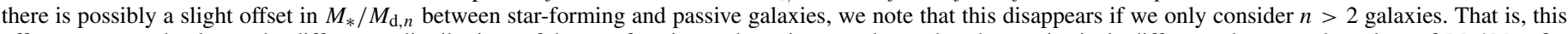

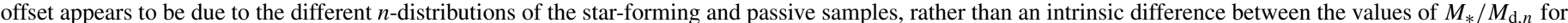

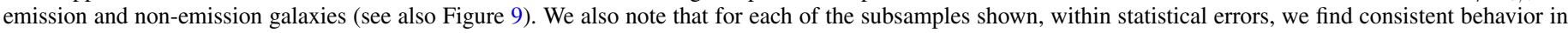

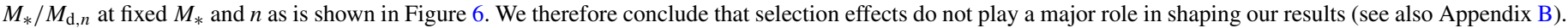

(A color version of this figure is available in the online journal.)

emission and non-emission subsamples, rather than any difference in $M_{*} / M_{\mathrm{d}, n}$ intrinsically related to galaxy activity (see also Section 5.3).

That is, our results do not appear to be strongly influenced by the relative numbers of central/satellite galaxies or of non/emission galaxies in our sample. The above analysis does suggest, however, that the measured relation between $M_{*}$ and $M_{\mathrm{d}, n}$ is sensitive to the joint $M_{*}-n$ distribution within the sample. This is a direct consequence of the fact that $M_{*} / M_{\mathrm{d}, n}$ depends on both mass and Sérsic index (Figure 6). We have considered biases associated with the mass distribution in Section 4.1. But note that if, at fixed mass, the distribution of $n$ within the G09 sample differs significantly from the "true" distribution for a general field population, then we may therefore find a very different slope for the $M_{*} / M_{\mathrm{d}, n}$ relation.
For this reason, in Appendix B, we repeat our $M_{*}-M_{\mathrm{d}, n}$ comparisons for a more general sample of $0.035<z<0.08$ field galaxies, using the structural parameters given in the NYU VAGC. ${ }^{12}$ The best-fit logarithmic slope of the $M_{*}-M_{\mathrm{d}, n}$ relation for $0.035<z<0.08$ field galaxies is $a=0.91 \pm 0.003$,

\footnotetext{
12 As we have already remarked, the VAGC Sérsic fits have been shown to suffer from systematic errors arising from background oversubtraction. But, as we also show in Appendix B, it turns out that the ratio $M_{*} / M_{\mathrm{d}, n}$ is extremely robust to random or systematic errors in the fitting of structural parameters, so long as the apparent magnitude, effective radius, and Sérsic index are derived consistently, and the term $K_{V}(n)$ is included in the definition of the dynamical mass (see also Section 6). Further, we find very similar $M_{*}-M_{\mathrm{d}, n}$ relations for the G09 sample using either VAGC or the G09 values for the structural parameters. Any large differences in the measured $M_{*}-M_{\mathrm{d}, n}$ relation between the G09 sample and the general field sample would therefore necessarily be a product of selection effects.
} 

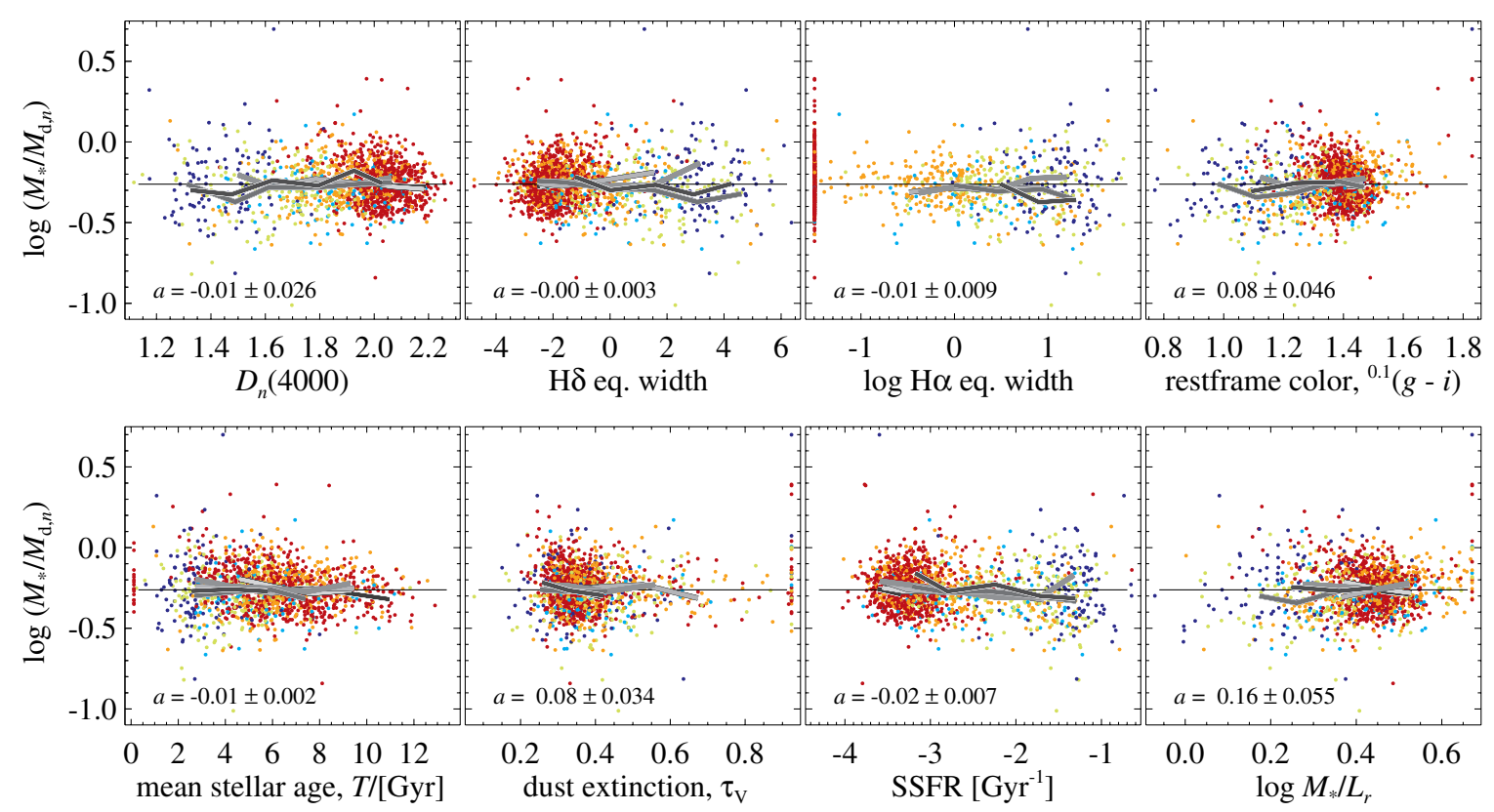

Figure 9. Stellar population-dependent effects? Each panel plots $M_{*} / M_{\mathrm{d}, n}$ as a function of a stellar population diagnostic. The upper panels show directly observed quantities; the lower panels show stellar population parameters derived from synthetic stellar population modeling. In these panels, the mean stellar age shown is that given by Kauffmann et al. (2003a), which are based on spectra, and are thus formally independent of $M_{*} / L$. The dust extinction, specific star formation rates, and $M_{*} / L$ s are all self-consistently derived from the same SED fits, as described in Section 2.3 . Within each panel, individual points are color-coded according to spectral classifications; specifically: star-forming (blue), low S/N star-forming (cyan), composite (yellow), AGN-dominated (orange), and "unclassifiable" (i.e., non-emission; red) galaxies. Within each panel, the black points with error bars show the median and 16/84 percentiles of $M_{*} / M_{\mathrm{d}, n}$ in bins. We see no statistically significant systematic differences in $M_{*} / M_{\mathrm{d}, n}$ for galaxies with different stellar populations or star formation histories. This argues against there being major problems with the stellar population models used to estimate $M_{*} / L$. At $99 \%$ confidence, these results suggest that any differential biases as a function of the parameters shown is $\lesssim 0.12 \operatorname{dex}(\approx 40 \%)$.

(A color version of this figure is available in the online journal.)

compared to $a=0.92 \pm 0.009$ for the G09 sample. This suggests that the G09 sample is not grossly biased in terms of the distribution of $n$ at fixed mass. (Here again, it is significant that G09 have found that, at fixed mass, there are no structural differences between satellite and central galaxies.)

To summarize the results of this section, then, separate analysis of central/satellite and non-/emission galaxies suggest that our results are not strongly affected by selection effects associated with these properties. Furthermore, although the observed slope of the $M_{*} / M_{\mathrm{d}, n}$ relation is in principle sensitive to the joint $M_{*}-n$ distribution within the sample, we find very little difference between the G09 sample and a more general field galaxy sample. Finally, we stress that we do not find any evidence that selection effects have an important impact on the results shown in Figure 6; i.e., the observation that the ratio $M_{*} / M_{\mathrm{d}, n}$ depends on both $n$ (at fixed $M_{*}$ ) and on $M_{*}$ (at fixed $n$ ).

\subsection{Looking for Biases in the Stellar Mass-to-Light Ratio Estimates}

As we have stated in Section 1, our primary motivation for comparing stellar and dynamical mass estimates is to validate the stellar mass estimates. We explore this issue in Figure 9. In each panel of this figure, we plot the ratio $M_{*} / M_{\mathrm{d}, n}$ as a function of a different property of the stellar population. The solid lines in each panel show the median relation for the same stellar mass bins shown in Figures 3 and 6. The upper panels plot $M_{*} / M_{\mathrm{d}, n}$ as a function of a direct observable; the lower panels plot $M_{*} / M_{\mathrm{d}, n}$ as a function of a derived property. Note that the stellar mass estimates we have used were derived from the ugriz photometry, rather than spectra. The measured values of $M_{*} / M_{\mathrm{d}, n}$ are thus formally independent of the three spectral measurements shown in the top panels. Further, note that the age estimate that we show is taken from Kauffmann et al. (2003a); these values are also derived from the spectra. The dust obscuration and SSFRs shown come from the SED fits used to derive $M_{*} / L$; these values are thus self-consistently derived.

We have color-coded the data in Figure 9 according to their spectral classification as given by Brinchmann et al. (2004), viz., star forming (blue); low S/N star forming (cyan); composite (yellow), AGN (orange), and non-emission (red). In general, we see little if any differences in the values of $M_{*} / M_{\mathrm{d}, n}$ for different stellar populations. There are no obviously large differences between the mean values of $M_{*} / M_{\mathrm{d}, n}$ for AGN hosts, starforming galaxies, or non-emission galaxies.

Within each panel, we give the gradient of the best-fit line for the whole sample. These values are all statistically consistent with zero. The only possible exceptions to this rule are the gradients in $M_{*} / M_{\mathrm{d}, n}$ as a function of $\mathrm{H} \alpha \mathrm{EW}$ and as a function of $M_{*} / L_{r}$, both of which are non-zero at the $\sim 3 \sigma$ level. Just as there is little if any trend for the sample as a whole, there are no statistically significant trends for any of the individual mass bins.

We can quantify the degree of correspondence between stellar and dynamical mass estimates by considering the differential bias between galaxies over the range of each of the properties shown in Figure 9. Looking at the median relations shown for each mass bin suggests that the magnitude of such differential biases are at most 0.2 dex. We can obtain similar estimates for the sample as a whole using the fit parameters given in each panel of Figure 9. Taking the $3 \sigma$ statistical limits on the slopes of these relations, we find that the differential effects across 
the full range of the sample are $\lesssim 0.1$ dex for $D_{n}(4000), \mathrm{H} \delta$ $\mathrm{EW}$, and rest-frame color, and $\lesssim 0.15$ dex for $\mathrm{H} \alpha \mathrm{EW}$, age, dust extinction, SSFR, and $M_{*} / L$.

The same is not true using the simple estimate of dynamical mass, $\tilde{M}_{\mathrm{d}}$, in place of $M_{\mathrm{d}, n}$. As might be expected from comparing Figures 1 and 4 , we find that the $M_{*} / \tilde{M}_{\mathrm{d}}$ relations for different mass bins are largely parallel, but significantly offset from one another. Then, because of correlations between mass and activity, we also find significant gradients in the $M_{*} / \tilde{M}_{\mathrm{d}}$ relations for the sample as a whole, typically at the 10-20 $\sigma$ level. The size of differential biases as a function of all of $D_{n}(4000)$, $\mathrm{H} \delta \mathrm{EW}, \mathrm{H} \alpha \mathrm{EW}$, age, and SSFR is on the order 0.2-0.4 dex. We note in particular that the relatively strong gradient in $\tilde{M}_{\mathrm{d}} / M_{*}$ with $\mathrm{H} \alpha \mathrm{EW}$ noticed by Drory et al. (2004) disappears when we use the structure-corrected dynamical mass estimator, $M_{\mathrm{d}, n}$, in place of the simple estimate $\tilde{M}_{\mathrm{d}}$; this apparent bias seems to be more closely linked to structure than to $\mathrm{H} \alpha$ emission per se. We thus find a very good correspondence between $M_{*}$ and $M_{\mathrm{d}, n}$ for galaxies in the G09 sample, but only provided we account for structural and dynamical non-homology.

\subsection{Color-M/L Relations}

Bell \& de Jong (2001) have shown that $M_{*} / L$ and color are strongly correlated in both the optical and the NIR. This implies that a single color is enough to make a reasonable estimate of $M_{*} / L$ (see also, e.g., Longhetti \& Saracco 2009; Gallazzi $\&$ Bell 2009). These kinds of color relations have since been widely used for high-redshift studies. In Taylor et al. (2010), we have shown that the values of $M_{*} / L$ that we use here correlate very strongly with ${ }^{0.1}(g-i)$ color, viz.,

$$
\log M_{*} / L_{i}=-0.82+0.83 \times \times^{0.1}(g-i) .
$$

(In this expression, $L_{i}$ should be understood as referring to the absolute luminosity in the ${ }^{0.1} i$-band filter; that is, the $i$-band filter redshifted to $z=0.1$. The absolute magnitude of the Sun in the ${ }^{0.1} i$ band is 4.58). The scatter around this relation is just 0.03 dex. If we use this relation to predict $M_{*} / L$ for galaxies in the G09 sample, we again find very good correspondence between $M_{*}$ and $M_{\mathrm{d}, n}$-in fact, the rms scatter in $M_{*} / M_{\mathrm{d}, n}$ is unchanged.

That is, at least from the point of view of consistency between $M_{*}$ and $M_{\mathrm{d}, n}$, it would seem that $M_{*} / L$ s estimated on the basis of a single color are not significantly worse than estimates based on full SED fits. This is significant because the SEDs that were used to derive the $M_{*} / L$ s were corrected for emission lines using the SDSS spectra. This kind of correction is not practical for, for example, high-redshift studies. Further, we have repeated our analysis using the $M_{*} / L s$ derived from the SDSS spectra by Kauffmann et al. (2003a), and find similarly good agreement between $M_{*}$ and $M_{\mathrm{d}, n}$ : at least on average, it would appear that color-derived $M_{*} / L \mathrm{~s}$ are just as good as those derived from optical spectroscopy (see also Gallazzi \& Bell 2009).

As can be seen in Figure 9, we find no statistically significant trend in $M_{*} / M_{\mathrm{d}, n}$ with ${ }^{0.1}(g-i)$ color. Given the existence of a relation between $M_{*} / L$ and color, this fact implies a relation between $M_{\mathrm{d}, n} / L$ and color. Using the color $-M_{*} / L_{i}$ relation given above, the results shown in Figure 9 imply that

$$
\begin{aligned}
\log M_{\mathrm{d}, n} / L_{i}= & (-0.54 \pm 0.07) \\
& +(0.80 \pm 0.05) \times \times^{0.1}(g-i) .
\end{aligned}
$$

(Again, $L_{i}$ in this expression should be understood as referring to the ${ }^{0.1} i$ band.) For the galaxies in our sample, the scatter around this relation is 0.14 dex, almost exactly the same as the scatter around the $M_{*}-M_{\mathrm{d}, n}$ relation. That is, using only the ${ }^{0.1}(g-i)$ color, it is possible to predict the dynamical masses of the galaxies in our sample with a relative uncertainty of $\approx 40 \%$.

\section{DISCUSSION}

\section{1. (Non)-Homology and Dynamical Mass Estimation}

In retrospect, it is perhaps unsurprising that $\tilde{M}_{\mathrm{d}}$ is not a good estimate of dynamical mass. By using a Sérsic-fit size and total magnitude measurements we have allowed for structural nonhomology in our definition of $\tilde{M}_{\mathrm{d}}$, while at the same time, by using $k=4$ for all galaxies, we have assumed dynamical homology. In this sense, the definition of $\tilde{M}_{\mathrm{d}}$ is not internally consistent.

On the other hand, it is remarkable that the SDSS model sizes can be used to obtain a reasonably good dynamical mass estimate, under the assumption of both structural and dynamical homology. As we argue in Appendix B, the $n$-dependence of $K_{V}(n)$ and the covariance between $n$ and the combination $R_{\mathrm{e}} / L$ are very nearly equal and opposite to one another. This leaves $M_{\mathrm{d}, n} / L \propto K_{V}(n) \sigma^{2} R_{\mathrm{e}} / L$ remarkably insensitive to errors in the size and total magnitude measurements, provided that (1) $m_{\mathrm{tot}}$, $\Theta_{\mathrm{e}}$, and $n$ are self-consistently derived and (2) $M_{\mathrm{d}, n}$ is derived using the appropriate value of $n$ to calculate the dynamics term $k$ or $K_{V}(n)$. This means that, for example, assuming a De Vaucouleurs profile (i.e., $n=4$ ) to derive $m_{r}$ and $\Theta_{\mathrm{e}}$ for all galaxies ensures that $\tilde{M}_{\mathrm{d}} / L \approx M_{\mathrm{d}, n} / L$, provided that the appropriate value of $K_{V}$ is used, viz., $k \approx K_{V}(n=4)=4.62$. This point is amply demonstrated in Figure 11 . That is, $M_{\mathrm{d}, n} / L$ appears to be insensitive to errors or assumptions in $n$, so long as everything is consistently derived self-consistently.

\section{2. $K_{V}(n)$ and Dynamical Mass Estimation}

The inclusion of the term $K_{V}(n)$ makes the structurecorrected dynamical mass estimator $M_{\mathrm{d}, n}$ explicitly model dependent. ${ }^{13}$ Further, this prescription for $K_{V}(n)$ has been derived under very simple and idealized assumptions (viz., a single component, spherical, and dynamically isotropic distribution), and so can only be regarded as approximate.

That said, more sophisticated dynamical models can give an indication as to how large these effects might be. For the case of anisotropy, the effects on the value of $K_{V}(n)$ are on the order of $\lesssim 0.1$ dex, and become less important for larger $n$ (see, e.g., Ciotti \& Lanzoni 2001). Further, Bertin et al. (2002) argue that the galaxy dynamics (or, more accurately, the value of $K_{V}$ ) close to the galaxy center are in principle rather sensitive to the precise shape of the total mass distribution. Their results suggest that this effect is on the order of $\lesssim 0.1$ dex (see their Figure D.1).

In order to probe the dependence of our conclusions on the assumed form of $K_{V}(n)$, we have also tried using an alternate prescription for $K_{V}(n)$, given by Cappellari et al. (2006; see their Equation (20)). The main difference between this prescription and the one given in Equation (3) is that it is phrased in terms of the observed velocity dispersion within the effective radius, $\sigma_{\mathrm{e}}$, rather than the central velocity dispersion $\sigma_{0}$. This prescription thus has a different dependence on dynamical isotropy and the dark matter profile, and so provides an indirect means of probing the importance of these effects. Using the Cappellari et al. (2006)

\footnotetext{
13 By the same token, the simple dynamical mass estimator $\tilde{M}_{\mathrm{d}}$ is also model
} dependent, inasmuch as it assumes homology, which is patently wrong. 
prescription, we find qualitatively and quantitatively similar results: we find that $M_{*} \propto M_{\mathrm{d}, n}^{0.85}$; we still see that $M_{*} / M_{\mathrm{d}, n}$ depends on $M_{*}$ at fixed $n$, and on $n$ at fixed $M_{*}$ (although this dependence is somewhat shallower); we see no trends in $M_{*} / M_{\mathrm{d}, n}$ with apparent magnitude or redshift, but a weak trend with observed size; and, at fixed mass, we see no statistically significant trends in $M_{*} / L$ with stellar population parameters.

That is, while it is virtually certain that the model used to derive the prescription for $K_{V}(n)$ given in Equation (3) is wrong in several important respects, it seems unlikely that accounting for those effects that are ignored in the model would have a drastic effect on our results and conclusions. As we have repeatedly stressed, detailed dynamical modeling is necessary better constrain the "true" values of $K_{V}$ for individual galaxies.

\subsection{Comparison to Previous Studies}

\subsubsection{Comparison to Other SDSS Studies}

In comparison to other studies of the relation between stellar and dynamical mass estimates based on SDSS data, we find considerably less variation in $M_{*} / M_{\mathrm{d}, n}$ with mass. In the case of Drory et al. (2004), this difference is simply due to the fact that we account for non-homology in the derivation of $M_{\mathrm{d}, n}$; using the simple mass estimate $\tilde{M}_{\mathrm{d}}$, we have verified that we are able to reproduce their results. In the case of Gallazzi et al. (2006), there is the additional complication that they use qualitatively different measures of total flux and size: specifically, the Petrosian magnitudes and half-light radii given in the basic SDSS catalog, which are derived directly from the observed curves of growth. Again, we have verified that we can reproduce their results using the same measurements.

Gallazzi et al. (2006) also split their galaxy sample into bins of Sérsic index, and find similar slopes to the $M_{*}-\tilde{M}_{\mathrm{d}}$ relation for each subsample; they find that the logarithmic slope of the relation varies from 0.847 to 0.801 between $n=3$ and $n=5.5$. The size of this variation is entirely consistent with the results we have shown in Figure 3. They use this fact to argue that nonhomology does not have a significant impact on the slope of the global $M_{*}-M_{\mathrm{d}, n}$ relation. However, as we have also shown in Figure 3, while the $M_{*}-\tilde{M}_{\mathrm{d}}$ relations for each bin in $n$ are parallel, they are significantly offset from one another. It is through this offset, combined with a correlation between $M_{*}$ and $n$, that non-homology affects the slope of the $M_{*}-M_{\mathrm{d}, n}$ relation; Gallazzi et al. (2006) make no mention of such an offset. While our conclusions differ with those of Gallazzi et al. (2006), our results are thus not obviously inconsistent.

\subsubsection{Comparison to Detailed Dynamical Modeling Results}

Cappellari et al. (2006) have also argued against the idea that non-homology has an important impact on dynamical mass estimates. This argument was based on dynamical mass-tolight ratios derived from detailed two-dimensional and threedimensional modeling 25 structurally early-type galaxies from the SAURON sample. Cappellari et al. (2006) compared the dynamical mass-to-light ratios, so derived, to the simple virial mass estimator $\tilde{M}_{\mathrm{d}} / L \propto \sigma^{2} R_{\mathrm{DeV}} / L_{\mathrm{DeV}}$, where $R_{\mathrm{DeV}}$ and $L_{\mathrm{DeV}}$ were derived from De Vaucouleurs profile fits. They found no evidence for an $n$-dependent offset between these two quantities.

The fact that Cappellari et al. (2006) use De Vaucouleurs fit sizes and magnitudes is significant: as we have argued above and in Appendix B, when using De Vaucouleurs fits to derive size and magnitude measurements, it is appropriate to use a constant $k$ in the definition of $\tilde{M}_{\mathrm{d}}$. That is, by using De Vaucouleurs-fit sizes and masses to define $\left(\tilde{M}_{\mathrm{d}} / L\right)$, Cappellari et al. (2006) effectively guaranteed, almost by construction, that they would find no structure dependence when comparing the "simple" dynamical mass-to-light ratio to that derived from full dynamical modeling.

\subsubsection{Comparison to Fundamental Plane Studies}

As we have mentioned in Section 1, the fundamental plane can be thought of as measuring the variation in the dynamical massto-light ratios of early-type galaxies as a function of velocity dispersion, luminosity, or mass (see, e.g., Dressler et al. 1987; Jørgensen et al. 1996). How do our derived values of $M_{\mathrm{d}, n}$ compare to those derived from the fundamental plane? To address this question, we selected the non-emission galaxies from within the G09 sample with $n>2.5$. For these galaxies, we find $M_{\mathrm{d}, n} / L \propto \sigma^{0.88 \pm 0.06}$. For comparison, Jørgensen et al. (1996) find $\sigma^{2} R_{\operatorname{DeV}} / L_{\operatorname{DeV}} \propto \sigma^{0.86}$, where again, $R_{\operatorname{DeV}}$ and $L_{\mathrm{Dev}}$ have been derived via De Vaucouleurs fits. Again, the covariance between the fit values of $m_{r}, \Theta_{\mathrm{e}}$, and $n$ means that $\sigma^{2} R_{\mathrm{DeV}} / L_{\mathrm{DeV}} \approx M_{\mathrm{d}, n} / L$. Our structure-corrected dynamical mass estimates are thus in good agreement with those derived from the fundamental plane. (Parenthetically, we also note that Cappellari et al. (2006) found that their dynamical mass-to-light ratios derived from detailed dynamical fits scaled as $\sigma^{0.82}$, which is also consistent with our results.)

We have also considered how the dynamical-to-stellar mass ratio, $M_{\mathrm{d}, n} / M_{*}$ varies with $\sigma$ for this same sample of early-type galaxies: we find $M_{\mathrm{d}, n} / M_{*} \propto \sigma^{0.50 \pm 0.06}$. This would suggest that less than half of the tilt of the fundamental plane is due to variations in the mass-to-light ratios of early-type galaxies as a function of $\sigma$ (cf., e.g., Prugniel \& Simien 1996; Trujillo et al. 2004; Allanson et al. 2009). We present this result only for completeness; proper interpretation of this result requires much more detailed analysis, and is beyond the scope of this work.

We also note that both Prugniel \& Simien (1996) and Trujillo et al. (2004) have made a very similar argument for the importance of non-homology in estimating dynamical masses as we have made in Sections 3 and 4, based on dynamical mass-to-light ratios derived from the fundamental plane. Our analysis based on the correspondence between $M_{*}$ and $M_{\mathrm{d}, n}$ is complementary to theirs in two ways. First, their analyses were specific to early-type galaxies; we have thus extended their result to the general galaxy population. Secondly, both authors focused on $M_{\mathrm{d}} / L$, rather than $M_{*} / M_{\mathrm{d}, n}$; that is, neither of these authors considered the relation between galaxies' stellar and dynamical masses.

\subsection{Interpretation}

Turning now to the interpretation of our results, the remarkable consistency between stellar and dynamical mass estimates shows two things. First, it strongly suggests that the measurements of $M_{*}$ and $M_{\mathrm{d}, n}$ are both meaningful and relatively robust. In particular, our results indicate that it is possible to derive stellar mass estimates without strong differential biases as a function of age, dust, SSFR, or $M_{*} / L$, based only on broadband optical photometry (or indeed on a single optical color).

Second, it implies that intrinsic variations in the stellar-todynamical mass ratio (due to, e.g., variations in the dark-tostellar mass ratio, variations in the IMF, or dynamical differences beyond the simple non-homology considered here) as a function of stellar mass, galaxy structure, and star formation rate/history are either small, or conspire to leave the inferred values of 
$M_{*} / M_{\mathrm{d}, n}$ relatively unchanged. The rest of this section will be devoted to the discussion of this result.

Since $M_{\mathrm{d}, n}$ is an estimate of total mass, it can only be interpreted as an upper bound on the true stellar mass. Because we have no a priori means of separating out the relative contributions of luminous and non-luminous mass to $M_{\mathrm{d}, n}$, the relation between $M_{*}$ and $M_{\mathrm{d}, n}$ is complicated by degeneracies between the relative contributions of gas and dark matter, as well as uncertainties in the low-mass shape of the IMF.

The simplest way to interpret the nonlinearity of the $M_{*}-M_{\mathrm{d}, n}$ relation is as indicating a greater central dark matter fraction for higher mass galaxies, in qualitative agreement with theoretical expectations. Using simple arguments based on the observed dynamics of elliptical galaxies, Franx (1993) and Kochanek (1994) have argued that accounting for a dark matter halo implies $\left|\log M_{*}\right| M_{\mathrm{d}, n} \mid \sim 0.14-0.18$ dex. This would go a long way toward explaining the $\sim 0.23 \pm 0.03$ offset that we have observed.

We can estimate gas masses using the prescription given by Zhang et al. (2009). These authors have used a sample of relatively low-mass SDSS galaxies with literature $\mathrm{H}$ I masses to derive a prescription for $M_{\mathrm{Hi}} / M_{*}$ as a function of $(g-r)$ color and stellar surface density. Using this prescription to derive baryonic mass estimates, $M_{\mathrm{bar}}=M_{*}+M_{\mathrm{HI}}$, reduces the size of the offset between $M_{\mathrm{bar}}$ and $M_{\mathrm{d}, n}$ by 0.05 dex to -0.18 dex, and brings the logarithmic slope of the $M_{\mathrm{bar}} / M_{\mathrm{d}, n}$ relation to 0.95 . The fact that the Zhang et al. (2009) relation has been derived for very different galaxies to the ones we consider here means that this result should be interpreted with caution. Even so, it is striking that, taken together, the estimated contributions of $\mathrm{HI}$ and dark matter almost perfectly explain the observed offset between $M_{*}$ and $M_{\mathrm{d}, n}$, and imply only a mild trend in $M_{\mathrm{bar}} / M_{\mathrm{d}, n}$ with mass: $M_{\mathrm{bar}} / M_{\mathrm{d}, n} \propto M_{\mathrm{bar}}^{-0.05}$.

Then there is the matter of the IMF. The effect of adopting a Salpeter (1955) IMF rather than that of Chabrier (2003) would be approximately to scale all our values of $M_{*}$ up by 0.22 dex. For a linear $M_{*}-M_{\mathrm{d}, n}$ relation (which our data are only marginally consistent with), this would leave virtually no room for dark matter or gas in the centers of galaxies in our sample. For the slightly less-than-linear relation preferred by our data, this would imply that $M_{*}>M_{\mathrm{d}, n}$ for galaxies with $M_{*} \lesssim 10^{11} M_{\odot}$, which is logically inconsistent. Thus we can say that, at best, our results are only marginally consistent with a Salpeter (1955) IMF. Accounting for dark matter, our results are also weakly inconsistent with a "diet Salpeter" IMF, and completely consistent with a Kroupa (2001) or Chabrier (2003) IMF. (Parenthetically, we also note that the results shown in Figure 9 can also provide a weak constraint on variations in the IMF as a function of star formation rate/history.)

Finally, we note that the observed scatter around the $M_{*}-M_{\mathrm{d}, n}$ relation is rather small: just 0.13 dex. We argue in Appendix B that the ratio $M_{*} / M_{\mathrm{d}, n} \propto \sigma^{2} R_{\mathrm{e}} / L$ is remarkably insensitive to errors in the Sérsic-fit parameters, provided that they are consistently derived, and that dynamical non-homology is taken into account. This implies that the uncertainties in $M_{*} / M_{\mathrm{d}, n}$ are dominated by errors in the measurement of $\sigma_{0}$ and $M_{*} / L$. The mean formal uncertainty in $\sigma$ for our galaxy sample is 0.034 dex. We estimate the mean random error in $M_{*} / L$ to be on the order of $0.1 \mathrm{dex}$; this is the random scatter between the SED-fit $M_{*} / L \mathrm{~s}$ used here and the spectrally derived $M_{*} / L \mathrm{~s}$ given by Kauffmann et al. (2003a). ${ }^{14}$ Adding these errors in

\footnotetext{
${ }^{14}$ Note that this is almost certainly an underestimate of the "true" random
}

quadrature (i.e., neglecting correlations between $\sigma$ and $M_{*} / L$ at fixed $M_{*}$ ) produces an uncertainty in $M_{*} / M_{\mathrm{d}, n}$ of 0.12 dex. This would imply that, at fixed $M_{*}$, the intrinsic scatter in $M_{*} / M_{\mathrm{d}, n}$ is potentially very small indeed: $\lesssim 0.04$ dex.

\section{SUMMARY}

The central focus of this work has been the degree of consistency between stellar and dynamical mass estimates, based on the latest generation of data products from the SDSS. We have shown that structural differences in galaxy dynamics can have a large impact on the estimated values of dynamical mass, and so on the degree of correspondence between stellar and dynamical mass (Section 3; Figures 1 and 4). Provided that we account for structure-dependent differences in galaxy dynamics (using the term $K_{V}(n)$, as defined in Equations (2) and (3)), we find very good agreement between the inferred stellar and dynamical masses of galaxies within the G09 sample.

Our analysis is based on the carefully constructed satellite/ central galaxy sample of G09, making selection effects a major potential concern. However, we find no signs of major differences in the relation between $M_{*}$ and $M_{\mathrm{d}, n}$ for central/ satellite or non-/emission galaxies within the sample, suggesting that our results are not seriously affected by selection effects (Section 5.2; Figure 8). Moreover, we find qualitatively and quantitatively similar results analyzing a more general sample of $0.035<z<0.08$ galaxies, using the best-fit Sérsic parameters given in the NYU VAGC, or using the De Vaucouleurs/ exponential model fit parameters given in the basic SDSS cata$\log$ (Appendix B).

We find that the ratio $M_{*} / M_{\mathrm{d}, n}$ varies with both $M_{*}$ and $n$ (Sections 3.2 and 4.2; Figures 3 and 6). While the apparent $n$-dependence of $M_{*} / M_{\mathrm{d}, n}$ is sensitive to the assumed form of $K_{V}(n)$, changing $K_{V}(n)$ cannot affect the result that $M_{*} / M_{\mathrm{d}, n}$ varies with $M_{*}$ at fixed $n$. Without spatially resolved dynamical information for individual galaxies, however, we cannot determine whether the apparent mass dependence of $M_{*} / M_{\mathrm{d}, n}$ is caused by some mass-dependent difference in galaxy dynamics, rather than a genuine physical difference in the stellar-todynamical mass ratios of galaxies with different masses.

Similarly, while we have shown very good agreement between stellar and dynamical masses for SDSS galaxies, we cannot unambiguously prove that neither of these quantities suffers from systematic biases. On the other hand, using the Sérsicfit parameters given by G09, we do not see any systematic variation in $M_{*} / M_{\mathrm{d}, n}$ with observed properties like apparent magnitude, apparent size, or redshift (Section 5.1; Figure 7). This is not true if we use the Sérsic-fit parameters given in the NYU VAGC, which has been shown to suffer from systematic errors arising due to background oversubtraction. That is, we have the ability to detect these sorts of errors, and do not see evidence for such errors for our sample. Further, we do not see any signs of variation in $M_{*} / M_{\mathrm{d}, n}$ for galaxies with different stellar populations, or for galaxies in different states of activity (i.e.,AGN hosts, star-forming galaxies, or non-emission galaxies).

These results, together with the good general agreement between $M_{*}$ and $M_{\mathrm{d}, n}$ provide strong circumstantial evidence (but not proof beyond a reasonable doubt) that there are no

uncertainty in $M_{*} / L$. Including NIR data (where the stellar population models are the most uncertain), and properly accounting for propagation of uncertainties in stellar population models and the IMF, Conroy et al. (2009) argue that the uncertainties in $M_{*} / L$ are on the order of 0.3 dex. 

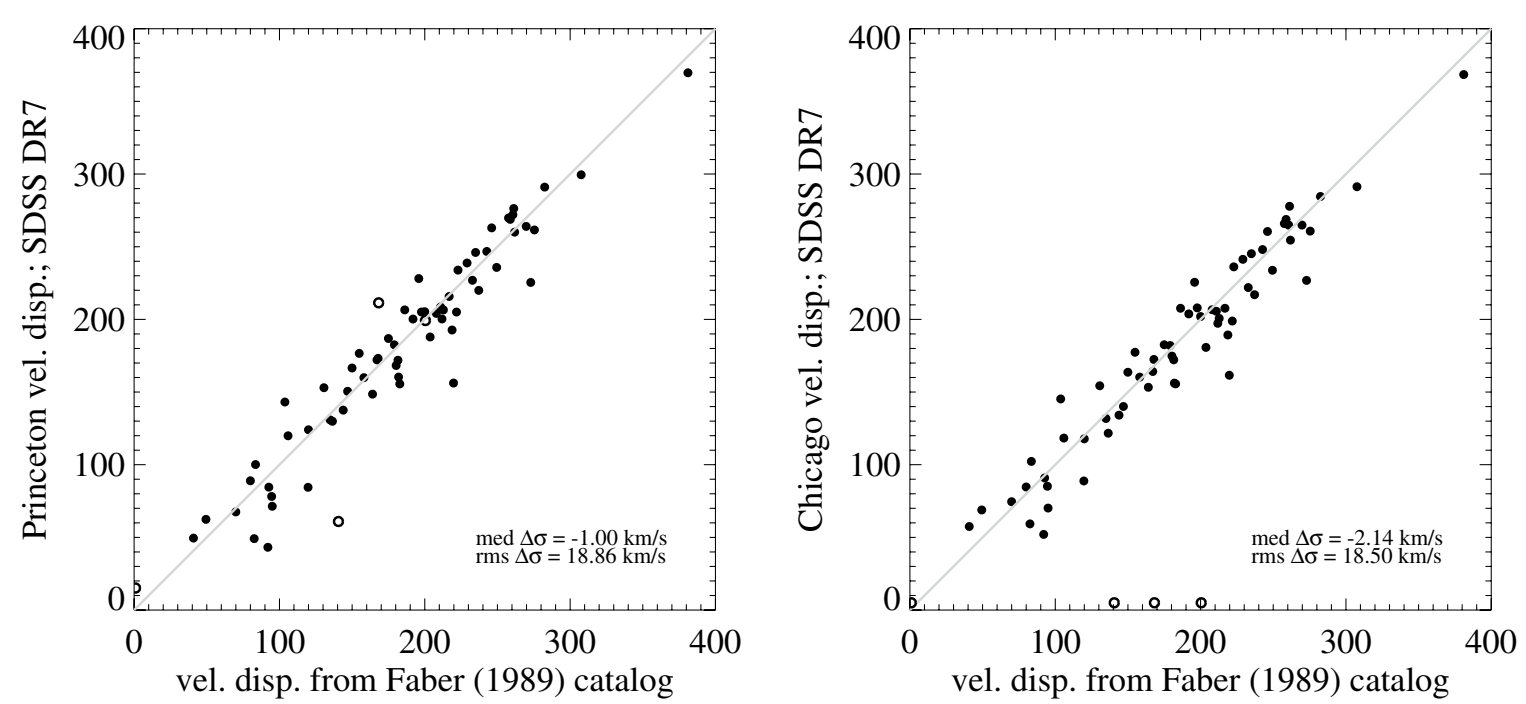

Figure 10. Comparison between the two different velocity dispersions measurements given in the SDSS DR7 catalog and those given by Faber et al. (1989). Bernardi (2007) have shown that the DR5 SDSS velocity dispersions suffered from systematic biases in comparison to those from the ENEAR sample as well as earlier SDSS releases. For this reason, the algorithms for estimating velocity dispersions from SDSS spectra were substantially revised for DR6 and later. In each panel of this figure, we compare one of the two SDSS velocity dispersion measurements to those in the "seven samurai" catalog (Faber et al. 1989). There are no signs of any systematic problems with the DR7 SDSS velocity dispersions. (Note however that the scatter in these comparisons is significantly higher than would be expected from the formal measurement uncertainties, which are on the order of $3.5 \mathrm{~km} \mathrm{~s}^{-1}$.)

serious systematic biases in the values of $M_{*}$ and $M_{\mathrm{d}, n}$ that we use here. This implies that the assumption of non-homology gives the wrong dynamical mass. Further, this suggests that there are no strong biases in the $M_{*} / L \mathrm{~s}$ we have used here: at $99 \%$ confidence, the consistency between $M_{*}$ and $M_{\mathrm{d}, n}$ implies any differential biases in the estimate of $M_{*} / L$ across a wide range of stellar populations are at the level of $\lesssim 0.12 \mathrm{dex}(\approx 40 \%)$.

This work was supported through grants by the Nederlandse Organisatie voor Wetenschappelijk Onderzoek (NWO), the Leids Kerkhoven-Bosscha Fonds (LKBF).

\section{APPENDIX A}

\section{VALIDATING THE SDSS DR7 VELOCITY DISPERSION MEASUREMENTS}

Bernardi (2007) showed that there was an inconsistency between the $\sigma-L$ relations for early-type galaxies derived using the early data release (EDR) and DR5 SDSS catalogs. Further, she was able to show that the cause for this discrepancy was systematic biases in the DR5 velocity dispersions: in comparison to literature values from HyperLeda, the DR5 measurements slightly but systematically overestimated the velocity dispersions of intrinsically low- $\sigma$ galaxies. For DR6+, partially in response to the findings of Bernardi (2007), the SDSS velocity dispersion pipelines were substantially revised. The new dispersions have been shown to agree well with the EDR velocity dispersions used by Bernardi et al. (2003a, 2003b), and thus, by implication, with the improved estimates for DR5 derived by Bernardi (2007). ${ }^{15}$

In this appendix, in order to validate the DR7 velocity dispersions, we present a comparison between the velocity dispersions given in the basic SDSS DR7 catalog to those given by Faber et al. (1989) for elliptical galaxies in their sample. The results of this comparison are shown in Figure 10. The left panel of this figure shows the comparison for the Princeton or

\footnotetext{
15 See http://www.sdss.org/dr7/algorithms/veldisp.html.
}

SpecBS values of $\sigma$; the right panel shows that for the Chicago or spectrold values of $\sigma$. Note that the Chicago algorithm only outputs values of $\sigma$ for those galaxies that are spectroscopically classified as being early type; the three Faber et al. (1989) galaxies at the bottom of the right-hand panel are not classified as being early type, and so are not given Chicago velocity dispersions.

Within both panels, we give the median and rms difference between the SDSS and Faber et al. (1989) velocity dispersion measurements. It is clear from this figure that neither of the DR7 velocity dispersions suffer from serious systematic biases in comparison to the Faber et al. (1989) measurements. We note, however, that the rms scatter, which is on the order of $19 \mathrm{~km} \mathrm{~s}^{-1}$, is considerably higher than the median formal measurement uncertainty given in the SDSS catalog, which is on the order of $3.5 \mathrm{~km} \mathrm{~s}^{-1}$ for the galaxies shown in Figure 10. That is, it seems that the formal uncertainties on the SDSS velocity dispersions significantly underestimates the true error, at least for the relatively bright galaxies shown here.

\section{APPENDIX B}

\section{SELECTION EFFECTS AND SYSTEMATIC BIASES: REPEATING OUR ANALYSIS FOR A GENERAL GALAXY SAMPLE}

As we have repeatedly stressed in the main text, the G09 sample that we analyze in the main text is heavily selected. In order to make sure that our conclusions are not unique to the G09 sample, in this appendix we repeat our analysis for a more general galaxy sample. For this exercise, we have selected $m_{\text {Pet, } r}<17.5$ galaxies with sciencePrimary spectra in the range $0.035<z<0.08$. As in our main analysis, we also require that the relative error on the velocity dispersion is less than $10 \%$, and that $\sigma_{\mathrm{ob}}>75 \mathrm{~km} \mathrm{~s}^{-1}$; these selections effectively limit the sample to $M_{*} \gtrsim 10^{10} M_{\odot}$. The additional incompleteness due to our velocity dispersion criteria is less than $10 \%$ for all $M_{*}>10^{10.3} M_{\odot}$ and $n \gtrsim 3$, but is significant for $n \lesssim 1$ at all masses. 

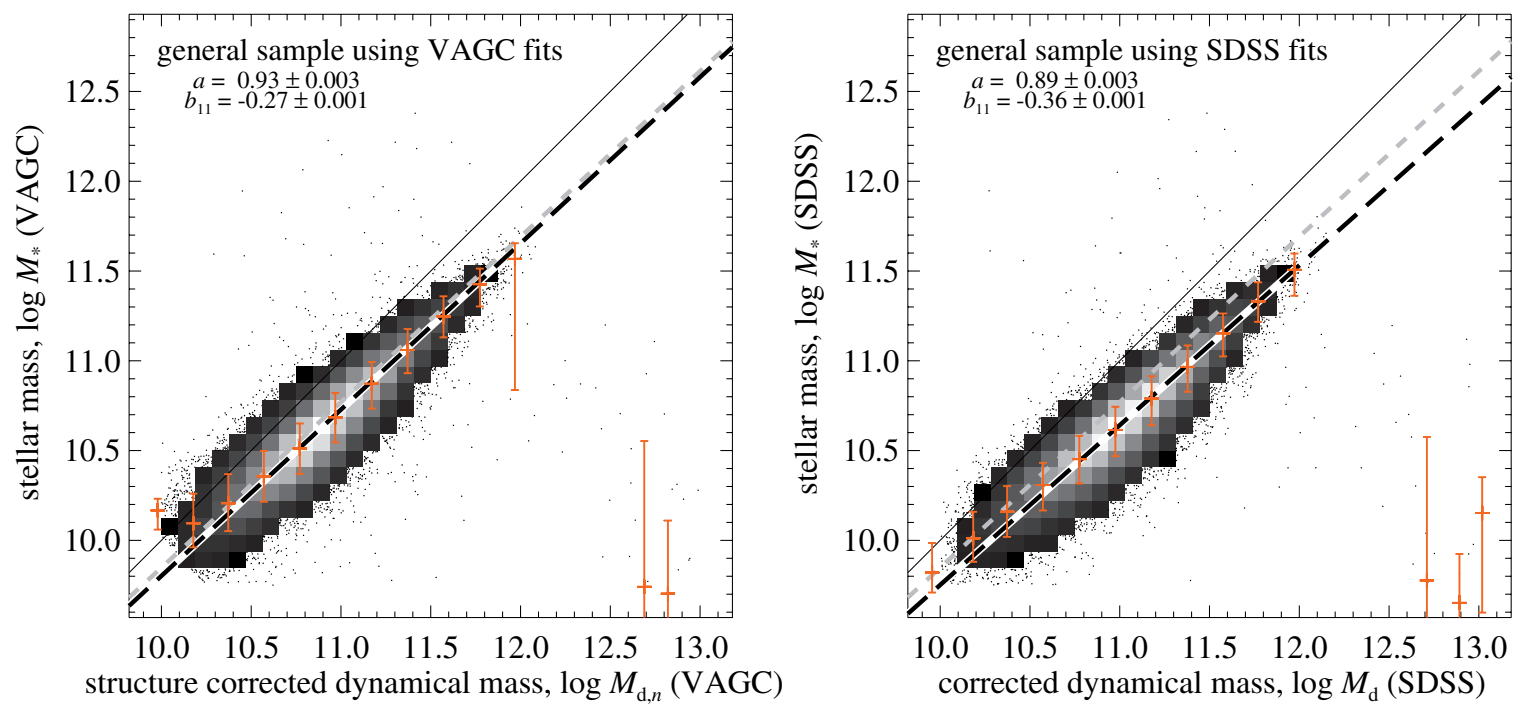

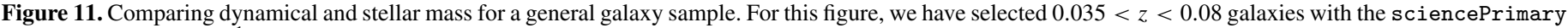

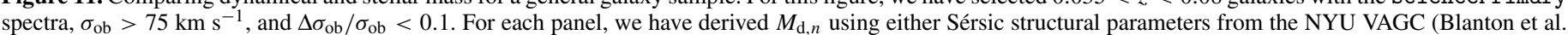



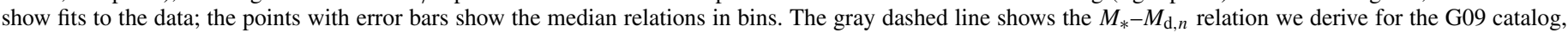
using their Sérsic structural parameters. The general $M_{*}-M_{\mathrm{d}, n}$ relation for field galaxies is very similar to the one we find for the heavily selected G09 sample.

(A color version of this figure is available in the online journal.)

There is one complicating factor in the comparison between this general field sample and the G09 sample that we discuss in the main text. For the field sample, we are forced to rely on either the De Vaucouleurs/exponential model fits provided in the basic SDSS catalog, or the Sérsic fits given in the NYU VAGC. (Recall that we need a measure of total magnitude to derive $M_{*}$, and both an effective radius and a Sérsic index measurement to derive $M_{\mathrm{d}, n}$.) Both of these sets of measurements have their faults. The SDSS model fits are overly simplistic in that they assume that $n$ is equal to either 1 or 4 ; this will clearly introduce systematic errors in the fit quantities as a function of (intrinsic) profile shape. The VAGC Sérsic fits are also known to suffer systematic errors (Blanton et al. 2005b), due to background oversubtraction (G09).

Bearing both these issues in mind, in Figure 11 we show the relation between $M_{*}$ and $M_{\mathrm{d}, n}$ for our general, field galaxy sample, using either the Sérsic-fit parameters from the NYU VAGC (left panel) or the model fit parameters from the basic SDSS catalog (right panel). Using either set of parameters, the results for this general sample agree really very well with what we have found for the G09 sample in Figure 4. Moreover, the two panels in Figure 11 agree remarkably well with one another, even despite the significant and very different systematic errors that each set of measurements suffers from.

How can this be? It turns out that the covariance between Sérsic parameters leaves ratio $M_{*} / M_{\mathrm{d}, n}$ is remarkably robust to both random and systematic errors in the Sérsic fits, provided $M_{\mathrm{d}, n}$ is calculated as per Equation (2). To illustrate this, let us compare the G09 and VAGC measurements. Although there are large differences in all three parameters individually, there are tight correlations between $\Delta n, \Delta m_{\text {tot }}$, and $\Delta R_{\mathrm{e}}$. (Here and in what follows the " $\Delta$ " implies the difference between the VAGC and Guo et al. (2009)-derived value, in the sense of VAGCminus-G09.) Now, $M_{*}$ scales directly with total flux; fitting to $\Delta M_{*}$ as a function of $\Delta n$, we find that $\Delta \log M_{*} \propto 0.04 \Delta n$, with an rms scatter in $\Delta \log M_{*}$ of 0.07 dex. $M_{\mathrm{d}, n}$, at least as defined in Equation (2), depends on both the effective radius and Sérsic index. Considering the change in $M_{\mathrm{d}, n}$ due to changes in size alone, we find $\Delta \log M_{\mathrm{d}, n} \propto 0.11 \Delta n$, with a scatter of $0.07 \mathrm{dex}$; for the effect due to changes in the Sérsic index alone, we find $\Delta \log M_{\mathrm{d}, n} \propto-0.06 \Delta n$, with an rms scatter of 0.03 dex. Taken together, the overall change in $M_{\mathrm{d}, n}$ scales with $\Delta n$ as $\Delta \log M_{\mathrm{d}, n} \propto 0.04 \Delta n$.

Thus, we see that the changes in $M_{*}$ and $M_{\mathrm{d}, n}$ thus have virtually the same dependence on $\Delta n$, leaving the ratio $M_{*} / M_{\mathrm{d}, n}$ virtually unchanged. Further, the scatter in $\Delta\left(M_{*} / M_{\mathrm{d}, n}\right)$ is just 0.04 dex. Using the basic SDSS model fits, while we find slightly stronger dependences with $\Delta n$, we still find that the ratio $M_{*} / M_{\mathrm{d}, n}$ remains very robust. We stress that the above argument only holds if we account for the dynamical effects of structure in the calculation of $M_{\mathrm{d}, n}$ : if we removed the $n$-dependence of $M_{\mathrm{d}, n}$ that enters via $K_{V}(n)$, then we would find that the ratio $\Delta \log \left(M_{*} / \tilde{M}_{\mathrm{d}}\right) \propto-0.06 \Delta n$, in agreement with the expectation from the analysis immediately above.

To explicitly demonstrate that the observed relation between $M_{*}$ and $M_{\mathrm{d}, n}$ is not particularly sensitive to the measurements used to derive the values of $M_{*}$ and $M_{\mathrm{d}, n}$, in Figure 12 we show the $M_{*}-M_{\mathrm{d}, n}$ relation for the G09 sample analyzed using structural parameters from the NYU VAGC (left panel) or from the SDSS catalog (right panel). In comparison to Figure 4, the slope of the $M_{*}-M_{\mathrm{d}, n}$ relation for the G09 sample is very similar using any of the three sets of structural parameters: 0.92 for the G09 fits, compared to 0.89 for the NYU VAGC fits, and 0.88 for the SDSS model fits. The normalization of the $M_{*}-M_{\mathrm{d}, n}$ relation is slightly more sensitive: at $M_{\mathrm{d}, n}=10^{11} M_{\odot}$, we find that $\Delta \log \left(M_{*} / M_{\mathrm{d}, n}\right)=-0.23,-0.24$, and -0.32 dex using the G09, VAGC, and SDSS fits, respectively.

In comparison to Figure 11, the results in Figure 12 also demonstrate that the $M_{*}-M_{\mathrm{d}, n}$ relation for the G09 is very similar to that for a more general field galaxy sample. For example, using structural parameters from the VAGC, the logarithmic slope and intercept of the $M_{*}-M_{\mathrm{d}, n}$ relation are $a=0.89$ and $b_{11}=-0.24$ for the G09 sample, compared to $a=0.91$ and $b_{11}=-0.27$ for the general galaxy sample.

In Figure 13, we show that galaxies in different states of activity follow very similar $M_{*}-M_{\mathrm{d}, n}$ relations. In this figure, 

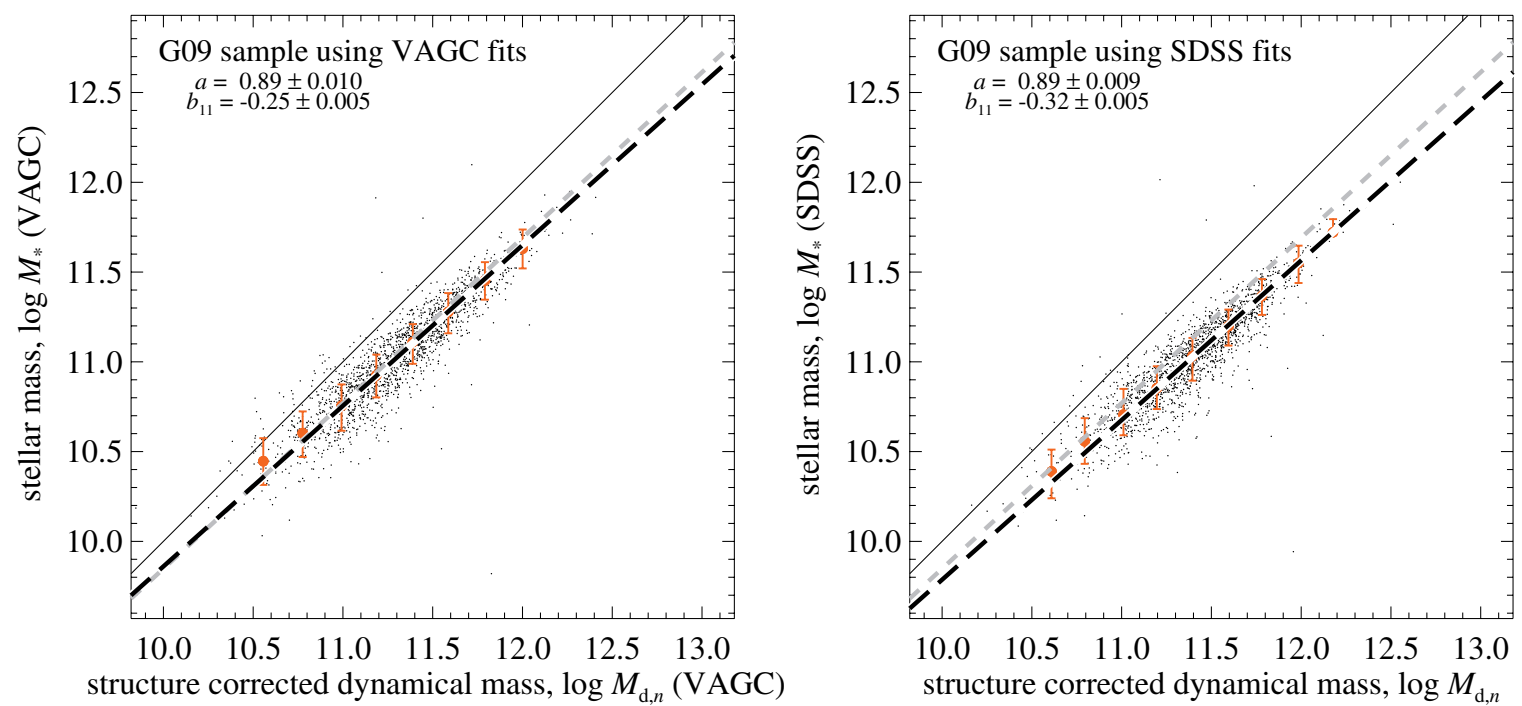

Figure 12. Comparing dynamical and stellar mass for the G09 sample, using structural fit parameters from the NYU VAGC or the basic SDSS catalog. All symbols and their meanings are directly analogous to Figures 4 and 11. In comparison to Figure 4, this figure differs only in that we have used the Sérsic-fit parameters from either the NYU VAGC (Blanton et al. 2005b) or the De Vaucouleurs/exponential model fit parameters from the basic SDSS catalog. The fact that these results agree very well with those shown in Figure 4 shows that the ratio $M_{*} / M_{\mathrm{d}, n}$ is not extremely sensitive to errors in the structural fit parameters. In comparison to Figure 11, the difference is that we only show galaxies in the G09 sample. The fact that the results in this figure agree very well with those shown in Figure 11 shows that sample selection effects do not play an important role in our results.

(A color version of this figure is available in the online journal.)
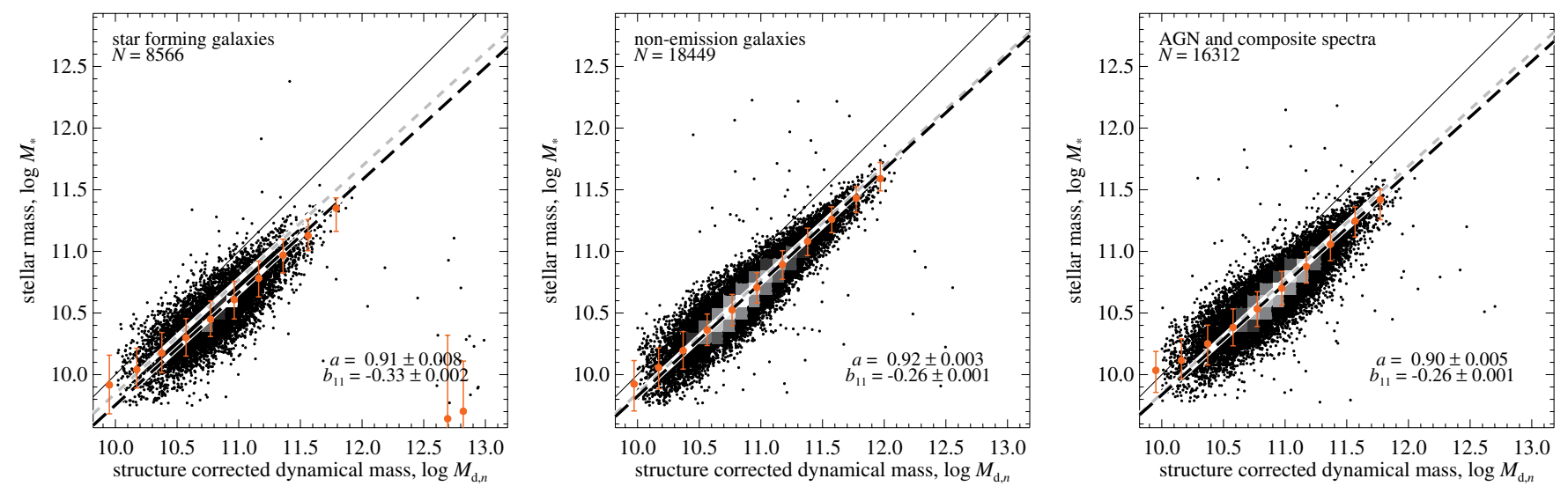

Figure 13. $M_{*}-M_{\mathrm{d}, n}$ relation for galaxies in different states of activity. Each panel of this figure shows the relation between $M_{*}$ and $M_{\mathrm{d}, n}$ for different subsamples of the general $0.035<z<0.08$ galaxy population, split according to their spectral classification, and analyzed using the Sérsic fits given in the NYU VAGC. From left to right, we show non-emission galaxies, star-forming galaxies, and AGN/composite spectra galaxies; the spectral classifications are those of Brinchmann et al (2004), which are based on the BPT diagram. In each panel, the heavy dashed line shows the best-fit $M_{*}-M_{\mathrm{d}, n}$ relation; for comparison, the gray short-dashed line shows the best-fit relation for the sample as a whole. While each subsample follows a similar $M_{*}-M_{\mathrm{d}, n}$ relation, there is an offset between the different relations, on the order of 0.07 dex. As for the G09 sample, the differences between the different subsamples disappear if we consider only $n>3$ galaxies. That is, these offsets appear to be due to the different distributions of $n$ within each subsample, rather than intrinsic differences in the values of the stellar-to-dynamical mass ratio for galaxies in different states of activity. This argues that selection effects play a major role in shaping our results.

(A color version of this figure is available in the online journal.)

we have split the general galaxy sample into non-emission, star forming, and AGN and composite spectra subsamples using the Brinchmann et al. (2004) spectral classification scheme described in Section 5.2. The logarithmic slopes of the $M_{*}-M_{\mathrm{d}, n}$ relation for each subsample agree with one another, as well as with that for the sample as a whole, to within a few percent. We do find that the $M_{*}-M_{\mathrm{d}, n}$ relation for the star-forming subsample is offset from that for the non-emission and AGN/ composite subsamples, at the level of 0.07 dex. However, as for the G09 sample (see Section 5.2), these small differences disappear if we consider only $n>2.5$ galaxies. We thus conclude that these differences are principally driven by the different distribution of $n$ values within the star-forming sample, rather than intrinsic differences in the stellar-to-dynamical mass ratios of star-forming galaxies.

Finally, in Figure 14, we separate out the $M_{*^{-}}$and $n$-dependences of $M_{*} / M_{\mathrm{d}, n}$ for the general galaxy sample, analyzed using the NYU VAGC Sérsic-fit parameters. Again, we find that the ratio $M_{*} / M_{\mathrm{d}, n}$ depends on both $M_{*}$ (at fixed $n$ ) and on $n$ (at fixed $M_{*}$ ). The results in this figure suggest that the mass dependence of $M_{*} / M_{\mathrm{d}, n}$ may flatten considerably for $n \lesssim 2$ and $10 \lesssim \log M_{*} / M_{\odot} \lesssim 10.5$ (i.e., below the mass limit of the G09 sample).

As we have noted above, there are significant differences in the values of $M_{*}$ and $n$ given by G09 and in the VAGC. These differences in $M_{*}$ and $n$ mean that the results in this figure are 

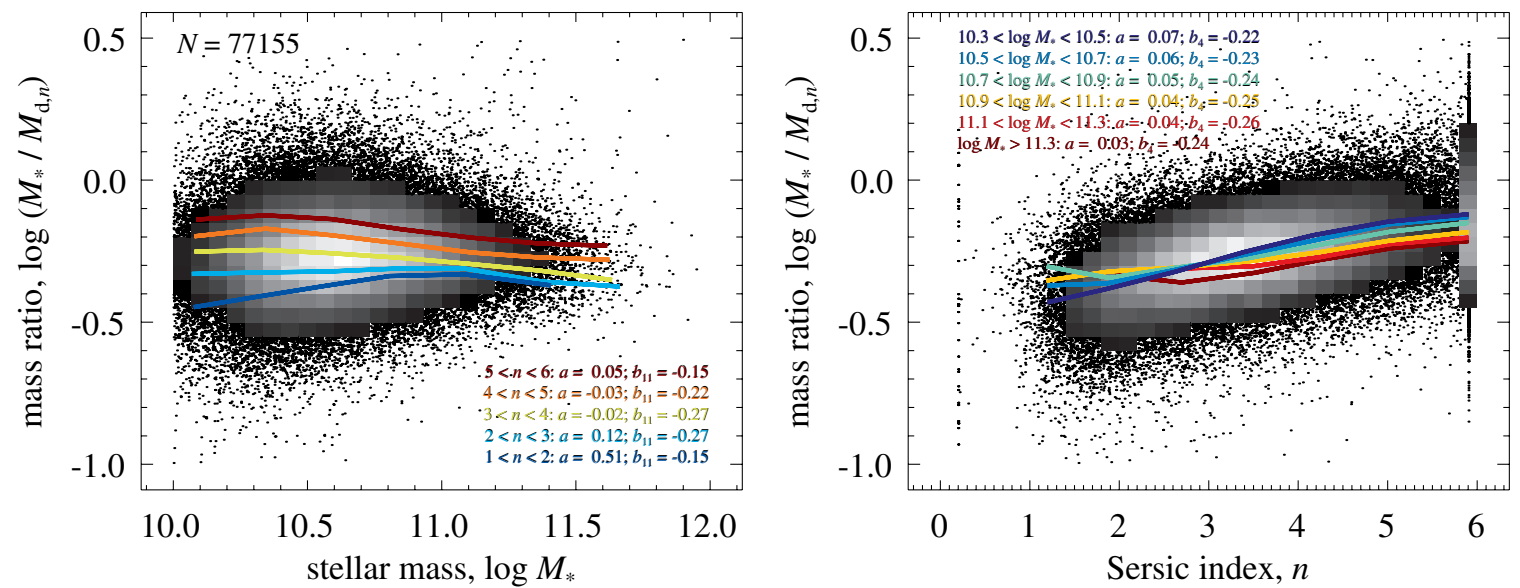

Figure 14. Separating out the mass and structure dependence of the mass ratio $M_{*} / M_{\mathrm{d}, n}$ of a field sample of $0.035<z<0.08$ galaxies, showing active and passive galaxies separately. In analogy to Figure 6, the colored lines in the left panels show the mass dependence of the mass ratio $M_{*} / M_{\mathrm{d}, n}$ in bins of Sérsic index; those in the right panel shows how $M_{*} / M_{\mathrm{d}, n}$ varies with $n$ in bins of $M_{*}$. The precise bins are given within each panel, along with the best-fit parameters for the relation for each bin. The results in this panel have been derived using the NYU VAGC Sérsic-fit parameters. In comparison to Figure 6, we see qualitatively similar behavior for $M_{*} / M_{\mathrm{d}, n}$ as a function of both $M_{*}$ and $n$ for the general galaxy sample as we do for the heavily selected G09 sample. Moreover, we point out that this is true for non-emission, star-forming, AGN, and composite spectra galaxies separately, as well as for the general sample as a whole. Because the NYU fits suffer systematic biases, there are quantitative differences in the results shown in this figure and those in Figure 6 . Despite these quantitative differences, the relatively weak dependence of $M_{*} / M_{\mathrm{d}, n}$ on $n$ for each bin in $M_{*}$ supports our main result; viz., that accounting for non-homology leads to reasonably good consistency between stellar and dynamical mass estimates.

(A color version of this figure is available in the online journal.)

not in quantitative agreement with those shown in Figure 14, even though both data sets show good agreement in the global $M_{*}-M_{\mathrm{d}, n}$ relation. In particular, the slope of the $M_{*} / M_{\mathrm{d}, n}-n$ relation at fixed $M_{*}$ is significantly steeper than we find for the G09 sample. This is at least partially due to the bias in the NYU values of $n$; the NYU values are systematically lower than the G09 values, which has the effect of steepening the $M_{*} / M_{\mathrm{d}, n}-n$ relation.

With these caveats, the main conclusion to be drawn from Figure 14 is that accounting for non-homology in the derivation of dynamical masses leads to considerably better consistency between $M_{*}$ and $M_{\mathrm{d}, n}$ (as a function of $n$, and at fixed $M_{*}$ ), in agreement with our findings in Section 4. Further, we note that we find similar and consistent behavior in $M_{*} / M_{\mathrm{d}, n}$ at fixed $M_{*}$ and $n$ for each of the three subsamples shown in Figure 13, in agreement with our conclusions above.

In summary, then, in this appendix we have demonstrated two things. First, we have shown that we find very similar results for the G09 sample, analyzed using the results of the Sérsic fits given by G09, and for a more general galaxy sample, analyzed using either the Sérsic fits given in the NYU VAGC or the De Vaucouleurs/exponential model fits given in the basic SDSS catalog. Second, we have shown that we find very similar results for the G09 sample analyzed using any of these three sets of structural parameters. The most important conclusion to be drawn from these results is that the results we have presented in the main text are not driven, nor particularly sensitive to, selection effects.

\section{REFERENCES}

Abazajian, K. N., et al. 2009, ApJS, 182, 543

Allanson, S. P., Hudson, M. J., Smith, R. J., \& Lucey, J. R. 2009, ApJ, 702, 1275 Baldwin, J. A., Phillips, M. M., \& Terlevich, R. 1981, PASP, 93, 5

Bell, E. F., \& de Jong, R. S. 2001, ApJ, 550, 212

Bell, E. F., McIntosh, D. H., Katz, N., \& Weinberg, M. D. 2003, ApJS, 149, 289

Bernardi, M. 2007, AJ, 133, 1954

Bernardi, M., et al. 2003a, AJ, 125, 1817
Bernardi, M., et al. 2003b, AJ, 125, 1849

Bertin, G., Ciotti, L., \& Del Principe, M. 2002, A\&A, 386, 149

Blanton, M. R., Eisenstein, D., Hogg, D. W., Schlegel, D. J., \& Brinkmann, J. 2005a, ApJ, 629, 143

Blanton, M. R., et al. 2005b, AJ, 129, 2562

Brinchmann, J., Charlot, S., White, S. D. M., Tremonti, C., Kauffmann, G., Heckman, T., \& Brinkmann, J. 2004, MNRAS, 351, 1151

Bruzual, G., \& Charlot, S. 2003, MNRAS, 344, 1000

Cappellari, M., et al. 2006, MNRAS, 366, 1126

Chabrier, G. 2003, ApJ, 586, L133

Ciotti, L., \& Lanzoni, B. 2001, A\&A, 321, 724

Conroy, C., Gunn, J. E., \& White, M. 2009, ApJ, 699, 486

Djorgovsky, S., \& Davis, M. 1987, ApJ, 313, 59

Dressler, A., Lynden-Bell, D., Burstein, D., Davies, R. L., Faber, S. M., Terlevich, R., \& Wegner, G. 1987, ApJ, 313, 42

Drory, N., Bender, R., \& Hopp, U. 2004, ApJ, 616, L106

Faber, S. M., Wegner, G., Burnstein, D., Davies, R. L., Dressler, A., Lynden-Bell, D., \& Terlevich, R. J. 1989, ApJS, 69, 763

Franx, M. 1993, in Galactic Bulges, ed. H. Dejonghe \& H. J. Habing (Dordrecht: Kluwer), 243

Franx, M., van Dokkum, P. G., Fórster-Schreiber, N. M., Wuyts, S., Labbé, I., \& Toft, S. 2008, ApJ, 688, 770

Gallazzi, A., \& Bell, E. F. 2009, ApJS, 185, 253

Gallazzi, A., Charlot, S., Brinchmann, J., \& White, S. D. M. 2006, MNRAS, 370,1106

Gallazzi, A., Charlot, S., Brinchmann, J., White, S. D. M., \& Tremonti, C. A. 2005, MNRAS, 362, 41

Graves, G. J., Faber, S. M., \& Schiavon, R. P. 2009, ApJ, 698, 1950

Guo, Y., et al. 2009, MNRAS, 398, 1129 (G09)

Jørgensen, I., Franx, M., \& Kjaergaard, P. 1995, MNRAS, 276, 1341

Jørgensen, I., Franx, M., \& Kjaergaard, P. 1996, MNRAS, 280, 167

Kannappan, S. J., \& Gawiser, E. 2007, ApJ, 657, L5

Kauffmann, G., Heckman, T. M., De Lucia, G., Brinchmann, J., Charlot, S., Tremonti, C., White, S. D. M., \& Brinkmann, J. 2006, MNRAS, 367, 1394 Kauffmann, G., et al. 2003a, MNRAS, 341, 33

Kauffmann, G., et al. 2003b, MNRAS, 341, 54

Kochanek, C. S. 1994, ApJ, 436, 56

Kroupa, P. 2001, MNRAS, 322, 231

La Barbera, F., Busarello, G., Merluzzi, P., de la Rosa, I. G., Coppola, G., \& Haynes, C. P. 2008, ApJ, 689, 913

Longhetti, M., \& Sarraco, P. 2009, MNRAS, 394, 774

Maraston, C. 2005, MNRAS, 362, 799

Padmanabhan, N., et al. 2004, New Astron., 9, 329

Peng, C. Y., Ho, L. C., Impey, C. D., \& Rix, H.-W. 2002, AJ, 124, 266

Prugniel, P. H., \& Simien, F. 1996, A\&A, 321, 111

Rettura, A., et al. 2006, A\&A, 658, 717 
Salpeter, E. E. 1955, ApJ, 121, 161

Shen, S., Mo, H. J., White, S. D. M., Blanton, M. R., Kauffmann, G., Voges, W., Brinkmann, J., \& Csabai, J. 2003, MNRAS, 343, 978

Sérsic, J.-L. 1963, Bol. Asoc. Argentina Astron., 6, 41

Sérsic, J.-L. 1968, Atlas de Galaxias Australes, Vol. 418 (Cordoba: Observatorio Astronomico), 617

Strauss, M. A., et al. 2002, AJ, 124, 1810

Taylor, E. N., Franx, M., Glazebrook, K., Brinchmann, J., van der Wel, A., \& van Dokkum, P. G. 2010, ApJ, 720, 723

Thakar, A. R., Szalay, A., Fekete, G., \& Gray, J. 2008, Comput. Sci. Eng., 10, 30
Trujillo, I., Burkert, A., \& Bell, E. F. 2004, ApJ, 600, L39

van der Wel, A., Franx, M., Wuyts, S., van Dokkum, P. G., Huang, J., Rix, H.-W., \& Illingworth, I. 2006, ApJ, 652, 97

Wolf, J., Martinex, G. D., Bullock, J. S., Kaplinghat, M., Geha, M., Muñoz, R. R., Simon, J. D., \& Avedo, F. F. 2010, MNRAS, 406, 1220

Yang, X., Mo, H. J., van den Bosch, F. C., Pasquali, A., Li, C., \& Barden, M. 2007, ApJ, 671, 153

Yip, C. W., et al. 2004, AJ, 128, 585

York, D. G., et al. 2000, AJ, 120, 1579

Zhang, W. E., Chang, L., Kauffmann, G., Hu, Z., Catinella, B., Shen, S., Guo, Q., \& Chang, R. 2009, MNRAS, 397, 1243 